LBNL - 43341

\title{
Attenuation and Fluctuations of Elastic Waves Due to Random Scattering From Inclusions
}

\author{
Valeri A. Korneev and Lane R. Johnson \\ Center for Computational Seismology, Lawrence Berkeley National Laboratory, \\ University of California, Berkeley, California 94720
}

May 1999

This study was supported by the Director, Office of the Energy Research, Office of Basic Energy Sciences,

Engineering and Geosciences Division of the U.S. Department of Energy under Contract No. DE-AC0376SF00098. Support was also provided by the Defense Special Weapons Agency under Grant No. DSWA-0197-1-0026. 


\section{DISCLAIMER}

This report was prepared as an account of work sponsored by an agency of the United States Government. Neither the United States Government nor any agency thereof, nor any of their employees, make any warranty, express or implied, or assumes any legal liability or responsibility for the accuracy, completeness, or usefulness of any information, apparatus, product, or process disclosed, or represents that its use would not infringe privately owned rights. Reference herein to any specific commercial product, process, or service by trade name, trademark, manufacturer, or otherwise does not necessarily constitute or imply its endorsement, recommendation, or favoring by the United States Government or any agency thereof. The views and opinions of authors expressed herein do not necessarily state or reflect those of the United States Government or any agency thereof. 


\section{DISCLAIMER}

Portions of this document may be illegible in electronic image products. Images are produced from the best available original document. 


\title{
Attenuation And Fluctuations of Elastic Waves \\ Due to Random Scattering From Inclusions
}

Valeri A. Korneev and Lane R. Johnson

\author{
Center for Computational Seismology, Lawrence Berkeley National Laboratory, \\ University of California, Berkeley, California 94720
}

\begin{abstract}
Exact solutions for elastic compressional and shear waves scattered from a homogeneous sphere are used to obtain formulas for velocity and attenuation of plane waves propagating through a layer of randomly distributed inclusions. Analytical expressions are obtained for the mean transmitted wave and fluctuations in its amplitude and phase over the complete range of frequencies. The size and contrast of the inclusions are arbitrary, but interactions between scatterers are not considered and the concentration of scatterers is assumed to be small. The results are compared with effective media expressions for velocities in the low frequency limit. The analytical solutions are also compared with numerical simulations and it is demonstrated that they satisfactorily explain the effects of scattering on both the mean and variance of the phase and the mean and variance of the attenuation for all frequencies. The need for spatial averaging of observational data and methods of interpreting such averaged data in terms of the material properties of the scattering medium are discussed.
\end{abstract}

Key words: waves, scattering, attenuation, sphere, diffraction, fluctuations

\section{Introduction}

The problem of elastic wave propagation through heterogeneous media is encountered in numerous disciplines. It has been particularly important in the discipline of seismology because the earth is 
heterogeneous on a broad range of scales, and so a variety of different approaches to this problem have been developed. For a medium heterogeneous in only one dimension the problem is essentially solved because exact solutions exist, although even in this situation the process of estimating and describing the heterogeneity in realistic applications commonly introduces approximations. For media heterogeneous in two or three dimensions the problem is much more profound, as it is necessary to combine approximate solutions of the wave equations with approximate descriptions of the media, and understanding when a particular set of approximations is valid is not a simple matter.

Because of the complexity of heterogeneity within the earth, it is typically modeled as a random medium in which the effects of the heterogeneity upon elastic waves is treated in a statistical sense. Chernov (1960) was one of the first to adopt this description of the medium and then assumed scalar wave propagation in obtaining results that have been used in a variety of applications. See for example Aki (1969, 1973, 1980), Wu (1982), Sato (1982a, 1982b), and Flatté and Wu (1988). Another common approach is to use the Born approximation in which it is assumed that the perturbations in the elastic wave parameters are linearly related to the perturbations in the medium. For examples of this approach see Knopoff and Hudson $(1964,1967)$, Hudson $(1968,1977)$, Sato (1984, 1990), Wu and Aki (1985a, 1985b), Wu (1989, 1994), and Li and Hudson $(1995,1997)$. While these various approaches to the problem of wave propagation in heterogeneous media have been successful in certain applications, they are accompanied by important limitations which can raise questions about the validity and generality of the results. Examples of such limitations are the lack of conversions between modes of propagation, the failure to conserve energy, or the inability to handle strong contrasts in material properties. It is the purpose of this paper to present a method of handling wave propagation in three-dimensional heterogeneous media which avoids some of these limitations. 
The basic approach followed in this paper is to treat the wave propagation process as a series of forward scattering problems. The medium is described as a random distribution of scatterers, where the size, material properties, and density of scatterers can vary. For the case where the scatterers are spherical and homogeneous, exact solutions for the single scattering process are used, but multiple scattering effects are only partly included. The use of these exact scattering solutions allows a complete treatment of mode conversions between $\mathrm{P}$ and $\mathrm{S}$ waves and arbitrary strong contrasts in material properties. They also provide the starting point for deriving low-frequency and high-frequency asymptotic solutions that can be compared with other approximate solutions.

\section{Scattering by a single inclusion}

It is necessary to first review some of the results from single scattering theory. These will be taken primarily from Korneev and Johnson (1993a, 1993b, 1996). Consider a homogeneous material with elastic parameters and density given by $\lambda_{2} \equiv \lambda, \mu_{2} \equiv \mu, \rho_{2}=\rho$. Velocities and wavenumbers for $\mathrm{P}$ and $\mathrm{S}$ waves, plus a velocity ratio, are defined by

$$
v_{p}=\sqrt{\frac{\lambda+2 \mu}{\rho}}, v_{s}=\sqrt{\frac{\mu}{\rho}}, k_{p}=\frac{\omega}{v_{p}}, k_{s}=\frac{\omega}{v_{s}}, \gamma=\frac{v_{s}}{v_{p}}
$$

where $\omega$ is angular frequency. At the origin of the coordinate system is a homogeneous inclusion having material properties $\lambda_{1}, \mu_{1}, \rho_{1}$, which are in general different from those of the surrounding material.

Consider a simple harmonic plane wave propagating in the direction of the positive $z$ axis of a rectangular coordinate system $(x, y, z)$.

$$
\tilde{\mathbf{u}}_{m 0}=\mathbf{u}_{m o} e^{i \omega t}, \quad \mathbf{u}_{m o}=\hat{\mathbf{u}}_{m} u_{m o}=\hat{\mathbf{u}}_{m} e^{-i k_{m} z}
$$

Here the index $m=p, s$ denotes either an incident $\mathrm{P}$ wave $\left(m=p, v_{m}=v_{p}, \hat{\mathbf{u}}_{m}=\hat{\mathbf{z}}\right)$ or an incident $\mathrm{S}$ wave, $\left(m=s, \quad v_{m}=v_{s}, \hat{\mathbf{u}}_{m}=\hat{\mathbf{x}}\right)$. 
The presence of the inclusion causes a disturbance of the incident wave which is described by the scattered field $\mathbf{u}_{s c}^{m}(r, \theta, \phi)$. Thus the total field outside of the scatterer has the form

$$
\mathbf{u}_{m}=\mathbf{u}_{m o}+\mathbf{u}_{s c}^{m}(r, \theta, \phi)
$$

Here a spherical coordinate system $(r, \theta, \phi)$ having the same origin as the rectangular coordinate system has been introduced. The scattered field may be expressed as a sum

$$
\mathbf{u}_{s c}^{m}(r, \theta, \phi)=\mathbf{u}_{m p}(r, \theta, \phi)+\mathbf{u}_{m s}(r, \theta, \phi)
$$

of scattered $\mathbf{P}$ and scattered $S$ waves, which in the far field approximation have the forms

$$
\begin{aligned}
& \mathbf{u}_{m p}(r, \theta, \phi)=u_{m o} \mathbf{A}_{m p}(\theta, \phi) \frac{e^{-i k_{p}(r-z)}}{r} \\
& \mathbf{u}_{m s}(r, \theta, \phi)=u_{m o} \mathbf{A}_{m s}(\theta, \phi) \frac{e^{-i k_{s}(r-z)}}{r}
\end{aligned}
$$

Note that in the far field the scattering function $\mathbf{A}_{m p}(\theta, \phi)$ is polarized along $\hat{\mathbf{r}}$ and orthogonal to $\mathbf{A}_{m s}(\theta, \phi)$ so that $\left(\mathbf{A}_{m p} \cdot \mathbf{A}_{m s}\right)=0$. Because of this, the change in the incident wave amplitude due to the interaction with a single inclusion can be expressed as

$$
\Delta u_{m}^{(1)}=\left(\mathbf{u}_{m}-\mathbf{u}_{m o}\right) \cdot \hat{\mathbf{u}}_{m}=u_{m o}\left[\mathbf{A}_{m m}(\theta, \phi)\right]_{c_{m}} \frac{e^{-i k_{m}(r-z)}}{r}
$$

where $c_{m}$ denotes the principal far field component associated with the wave index $m$. For the geometry described above, $c_{m}=z$ for an incident $\mathrm{P}$ wave and $c_{m}=x$ for an incident $\mathrm{S}$ wave.

We also need a result relating the total energy in the scattered field to the forward scattered amplitude having the polarization of the incident wave, known as a forward scattering theorem or an optical theorem. Let $F_{m o}$ be the energy flux per unit area averaged over one period and let $\Omega_{1}$ be a closed spherical surface of unit radius surrounding the inclusion. Then it is easy to show that the scattering cross sections related to the energy of the incident $P$ and $S$ waves are given by

$$
\begin{aligned}
\sigma_{p} & \equiv \frac{F_{s c}^{p}}{F_{p o}}=\int_{\Omega_{1}}\left(\left|\mathbf{A}_{p p}(\theta, \phi)\right|^{2}+\gamma\left|\mathbf{A}_{p s}(\theta, \phi)\right|^{2}\right) d \Omega_{1}=-\frac{4 \pi}{k_{p}} \operatorname{Im}\left\{\left[\mathbf{A}_{p p}(0,0)\right]_{z}\right\} \\
\sigma_{s} & \equiv \frac{F_{s c}^{s}}{F_{s o}}=\frac{1}{\gamma} \int_{\Omega_{1}}\left(\left|\mathbf{A}_{s p}(\theta, \phi)\right|^{2}+\gamma\left|\mathbf{A}_{s s}(\theta, \phi)\right|^{2}\right) d \Omega_{1}=-\frac{4 \pi}{k_{s}} \operatorname{Im}\left\{\left[\mathbf{A}_{s s}(0,0)\right]_{x}\right\}
\end{aligned}
$$




\section{Scattering by a layer of inclusions}

Consider a thin slab extending to infinity perpendicular to the $z$ axis between $z$ and $z+\Delta z$. Inclusions having the same size, shape, and elastic properties are uniformly distributed within this slab with a density of $N(z)$, the number of inclusions per unit volume (Figure 1). The average effect upon a plane wave propagating through this slab is obtained by summing up the effects of all the individual inclusions (equation (5))

$$
\begin{aligned}
\Delta \bar{u}_{m}(z+\Delta z)=\Delta z \int_{\infty}^{\infty} \int_{\infty}^{\infty} N(z) \Delta u_{m}^{(1)}(x, y, z) d x d y \\
=\Delta z N(z) \bar{u}_{m}(z) \int_{\infty}^{\infty} \int_{\infty}^{\infty}\left[\mathbf{A}_{m m}(\theta, \phi)\right]_{c_{m}} \frac{e^{-i k_{m}(r-z)}}{r} d x d y
\end{aligned}
$$

It is assumed in writing the above expression that the size and density of the inclusions is small enough so that scattering interactions between the inclusions can be neglected. Next we assume that the transmitted coherent plane wave is influenced only by the inclusions in a small cone about $\theta=0$ which coincides with a few central Fresnel zones (Hulst, 1957). Then, approximating $\mathbf{A}_{m m}(\theta, \phi)$ by $\mathbf{A}_{m m}(0,0)$ and using the parabolic approximation for the phase function $-k_{m} r$, we obtain

$$
\Delta \bar{u}_{m}(z+\Delta z)=-i \Delta z \frac{2 \pi}{k_{m}} N(z) A_{m}(z) \bar{u}_{m}(z)
$$

where

$$
A_{m}(z) \equiv\left[\mathbf{A}_{m m}(0,0)\right]_{c_{m}}
$$

is defined as the forward scattering coefficient at any value of $z$, In the limit as $\Delta z$ becomes small this becomes the differential equation

$$
\frac{d}{d z} \bar{u}_{m}(z)=-i \frac{2 \pi}{k_{m}} N(z) A_{m}(z) \bar{u}_{m}(z)
$$


This equation can be integrated for a layer having total thickness $Z$ to obtain the net effect upon a plane wave passing through a layer $(0 \leq z \leq Z)$ having inclusions distributed with a density $N(z)$

$$
\bar{u}_{m}(Z)=u_{m o} e^{-i \frac{2 \pi}{k_{m}} \int_{0}^{Z} N(z) A_{m}(z) d z}
$$

The result in equation (11) represents the average plane wave after it has propagated through a layer of thickness $Z$. It is sometimes referred to as the coherent part of the total field. A similar result was obtained by Groenenboom and Snieder (1995) for the two-dimensional scalar problem using the method of stationary phase.

The transimitted wave in equation (11) can also be expressed in the form (see Appendix C)

$$
\bar{u}_{m}(Z)=u_{m o} e^{i \bar{\phi}_{m}(Z)} e^{-\bar{q}_{m}(Z)}
$$

where the non-dimensional phase shift $\bar{\phi}_{m}$ and attenuation $\bar{q}_{m}$ are effective parameters which have the simple relationships

$$
\bar{\phi}_{m}(Z)=\omega \Delta t_{m}(Z), \quad \bar{q}_{m}(Z)=\frac{\alpha_{m}(Z)}{2} Z
$$

with an effective travel time deviation $\Delta t_{m}$ and an effective scattering attenuation coefficient $\alpha_{m}$. Then, comparing equations (11) and (12), it is clear that

$$
\bar{\phi}_{m}(Z)=-\frac{2 \pi}{k_{m}} \int_{0}^{Z} N(z) \operatorname{Re}\left\{A_{m}(z)\right\} d z
$$

and

$$
\bar{q}_{m}(Z)=-\frac{2 \pi}{k_{m}} \int_{0}^{Z} N(z) \operatorname{Im}\left\{A_{m}(z)\right\} d z
$$

Using the optical theorem of equation (6) we also have

$$
\bar{q}_{m}(Z)=\frac{1}{2} \int_{0}^{Z} N(z) \sigma_{m}(z) d z
$$

It is clear from equations (13) and (16) that a local attenuation coefficient $\alpha_{m}(z)$ can be defined by

$$
\alpha_{m}(z)=N(z) \sigma_{m}(z)
$$


From equations (13) and (14) the time shift of the transmitted wave is

$$
\Delta t_{m}(Z)=-\frac{2 \pi}{\omega k_{m}} \int_{0}^{Z} N(z) \operatorname{Re}\left\{A_{m}(z)\right\} d z
$$

This can be written as

$$
\Delta t_{m}(Z)=\overline{\Delta_{m}}(Z) \frac{Z}{v_{m}}
$$

where $\overline{\Delta_{m}}(Z)$ is the weighted average

$$
\overline{\Delta_{m}}(Z)=-\frac{2 \pi}{k_{m}^{2}} \frac{1}{Z} \int_{0}^{Z} N(z) \operatorname{Re}\left\{A_{m}(z)\right\} d z
$$

It follows that the effective velocity is given by

$$
v_{m}^{e f}=\frac{v_{m}}{1-\overline{\Delta_{m}}(Z)}
$$

Thus the time shift parameter of equation (20), which is essentially an average of the real part of the forward scattering function, translates directly into an effective velocity.

The results contained in equations (11) to (21) are generally true for any size of inclusion if the assumption of single scattering is justified. Note, however, that only scattering attenuation is included here, and intrinsic attenuation due to absorption of energy must be included separately.

\section{Attenuation and phase fluctuations}

In the previous section formulas for the average attenuation and phase shift caused by propagation through a layer containing randomly distributed inclusions were derived. Because of the randomness involved in this problem, it is to be expected that there would be fluctuations about these average values if a series of experiments were performed for different realizations of the inclusion distribution. In order to estimate these fluctuations it is necessary to consider the squared scattering effect $\Delta\left|u_{m}\right|^{2}$ given by

$$
\Delta\left|u_{m}\right|^{2}=\left|u_{m o}+\Delta u_{m}^{(1)}\right|^{2}-\left|u_{m o}\right|^{2}
$$




$$
\begin{aligned}
& =2 \operatorname{Re}\left\{u_{m o}^{*} \Delta u_{m}^{(1)}\right\}+\left|\Delta u_{m}^{(1)}\right|^{2} \\
& =2\left|u_{m o}\right|^{2} \operatorname{Re}\left\{\left[\mathbf{A}_{m m}(\theta, \phi)\right]_{c_{m}} \frac{e^{-i k_{m}(r-z)}}{r}\right\}+\left|\Delta u_{m}^{(1)}\right|^{2}
\end{aligned}
$$

The next step is to calculate the spatial average of this expression as the wave propagates through a thin slab of thickness $\Delta z$. The first term on the right presents no problem, as it is essentially the expression evaluated in equation (7). For the second term on the right we follow a procedure similar to that used for equation (7) and have

$$
\begin{array}{r}
\overline{\left|\Delta u_{m}(z+\Delta z)\right|^{2}}=\Delta z N(z) \int_{\infty}^{\infty} \int_{\infty}^{\infty}\left|\Delta u_{m}^{(1)}(x, y, z)\right|^{2} d x d y \\
=\Delta z N(z) \overline{\left|u_{m}(z)\right|^{2}} \int_{\infty}^{\infty} \int_{\infty}^{\infty}\left|\left[\mathbf{A}_{m m}(\theta, \phi)\right]_{c_{m}}\right|^{2} \frac{d x d y}{r^{2}}
\end{array}
$$

For the case where the scatterers are spheres of radius $R$, the results given in the Appendix A (equation $\mathrm{A}-5)$ for the Mie scattering regime $\left(k_{m} R \simeq 1\right.$ ) can be substituted into equation (22). Evaluations of the resulting integrals are given in Appendix B, and we have

$$
\overline{\left|\Delta u_{m}(z+\Delta z)\right|^{2}}=2 \pi \Delta z N(z) \overline{\left|u_{m}(z)\right|^{2}}\left|A_{m}(z)\right|^{2} G_{m}
$$

where $G_{m}$ is a non-dimensional function of frequency and elastic parameters. The complete expression for the averaged fluctuations is thus

$$
\Delta \overline{\left|u_{m}(z+\Delta z)\right|^{2}}=\Delta z N(z) \overline{\left|u_{m}(z)\right|^{2}}\left[\frac{4 \pi}{k_{m}} \operatorname{Im}\left\{A_{m}(z)\right\}+2 \pi\left|A_{m}(z)\right|^{2} G_{m}\right]
$$

which can also be written in terms of the scattering cross section of equation (6) as

$$
\Delta \overline{\left|u_{m}(z+\Delta z)\right|^{2}}=\Delta z N(z) \overline{\left|u_{m}(z)\right|^{2}}\left[-\sigma_{m}(z)+2 \pi\left|A_{m}(z)\right|^{2} G_{m}\right]
$$

In the low-frequency limit $k_{m} R \ll 1$ (Rayleigh scattering) equation (24) can be reduced to

$$
\Delta \overline{\left|u_{m}(z+\Delta z)\right|^{2}}=\Delta z N(z) \overline{\left|u_{m}(z)\right|^{2}}\left[-\sigma_{m}(z)+2 \pi\left|A_{m}(z)\right|^{2} G_{m}^{(0)}\right]
$$


with constant $\left|G_{m}^{(0)}\right|<1$. In the high-frequency limit $k_{m} R \gg 1$ it becomes

$$
\Delta \overline{\left|u_{m}(z+\Delta z)\right|^{2}}=\Delta z N(z) \overline{\left|u_{m}(z)\right|^{2}}\left[-\sigma_{m}(z)+\frac{9 \pi}{2 k_{m}^{2} R^{2}}\left|A_{m}(z)\right|^{2}\right]
$$

However, as pointed out in Appendix A, equation (A-6) should be used in place of equation (A-5) at high frequencies, and this leads to

$$
\Delta \overline{\left|u_{m}(z+\Delta z)\right|^{2}}=\Delta z N(z) \overline{\left|u_{m}(z)\right|^{2}}\left[-\sigma_{m}(z)+\frac{4 \pi}{k_{m}^{2} R^{2}}\left|A_{m}(z)\right|^{2}\right]
$$

Equations (26) and (27) differ by the insignificant factor 1.125 , which justifies the use of equation (24) for all frequencies.

Consider the general result of equation (24) and take the limit as $\Delta z \rightarrow 0$ to obtain

$$
\frac{d}{d z} \overline{\left|u_{m}(z)\right|^{2}}=N(z)\left[-\sigma_{m}(z)+2 \pi\left|A_{m}(z)\right|^{2} G_{m}\right] \overline{\left|u_{m}(z)\right|^{2}}
$$

Integrating this equation from $z=0$ to $z=Z$ yields

$$
\overline{\left|u_{m}(Z)\right|^{2}}=\left|u_{m o}\right|^{2} e^{-\int_{0}^{Z} N(z) \sigma_{m}(z) d z} e^{2 \pi \int_{0}^{Z} N(z)\left|A_{m}(z)\right|^{2} G_{m} d z}
$$

This result represents the fluctuations in the transmitted field after passing through a layer of arbitrary thickness $Z$.

The variance of the transmitted field is given by

$$
\operatorname{var}\left\{u_{m}(Z)\right\}=\overline{\left|u_{m}(Z)\right|^{2}}-\left|\bar{u}_{m}(Z)\right|^{2}
$$

and from equation (11) and (6)

$$
\left|\bar{u}_{m}(Z)\right|^{2}=\left|u_{m o}\right|^{2} e^{-\int_{0}^{Z} N(z) \sigma_{m}(z) d z}
$$

Thus

$$
\operatorname{var}\left\{u_{m}(Z)\right\}=\left|u_{m o}\right|^{2} e^{-\int_{0}^{Z} N(z) \sigma_{m}(z) d z}\left[e^{2 \pi \int_{0}^{Z} N(z)\left|A_{m}(z)\right|^{2} G_{m} d z}-1\right]
$$


This expression clearly shows the two competing effects that control the fluctuations in the transmitted field. The term in brackets, which represents the conversion of energy from the coherent field into random fluctuations, starts from zero when $Z=0$ and grows continuously as $Z$ increases. This growth is tempered by the decaying term in front of the brackets, representing the continuous loss of energy from the coherent field caused by the scattering. As is shown below, the combination of these two effects leads to a maximum in the fluctuations at a particular value of $Z$.

Note that another method of characterizing the relative size of the fluctuations is in terms of a coefficient of variation defined as

$$
C O V=\left[\frac{\operatorname{var}\left\{u_{m}(Z)\right\}}{\left|\bar{u}_{m}(Z)\right|^{2}}\right]^{1 / 2}=\left[e^{2 \pi \int_{0}^{Z} N(z)\left|A_{m}(z)\right|^{2} G_{m} d z}-1\right]^{1 / 2}
$$

This result shows that the relative size of the fluctuations grows monotonically with the distance of propagation.

It is shown in Appendix $\mathrm{C}$ that the average squared value of the phase shift of equation (14) and the attenuation of equation (15) are equal to each other and have the value

$$
\overline{\left|\phi_{m}(Z)\right|^{2}}=\overline{\left|q_{m}(Z)\right|^{2}}=\pi \int_{0}^{Z} N(z)\left|A_{m}(z)\right|^{2} G_{m} d z
$$

When the high frequency result of equation (27) is used in place of equation (24), the variance becomes

$$
\operatorname{var}\left\{u_{m}(Z)\right\}=\left|u_{m o}\right|^{2} e^{-\int_{0}^{Z} N(z) \sigma_{m}(z) d z}\left[e^{\frac{4 \pi}{k_{m}^{2}} \int_{0}^{Z} N(z) \frac{\left|A_{m}(z)\right|^{2}}{R^{2}} d z}-1\right]
$$

Numerical calculations at high frequencies reveal that in general

$$
\operatorname{Im}\left\{\left[\mathbf{A}_{m m}(0,0)\right]_{c_{m}}\right\} \gg \operatorname{Re}\left\{\left[\mathbf{A}_{m m}(0,0)\right]_{c_{m}}\right\}
$$

and therefore from equation (6) it follows that

$$
\left|A_{m}(z)\right|^{2} \approx\left|\operatorname{Im}\left\{\left[\mathbf{A}_{m m}(0,0)\right]_{c_{m}}\right\}\right|^{2}=\left(\frac{k_{m} \sigma_{m}(z)}{4 \pi}\right)^{2}
$$


This result is also consistent with formula (A-3). Substituting it into equation (35) results in

$$
\operatorname{var}\left\{u_{m}(Z)\right\}=\left|u_{m o}\right|^{2} e^{-\int_{0}^{Z} N(z) \sigma_{m}(z) d z}\left[e^{\frac{1}{4 \pi} \int_{0}^{Z} N(z) \frac{\sigma_{m}^{2}(z)}{R^{2}} d z}-1\right]
$$

A further simplification is possible if we use an asymptotic expression for the scattering cross-section $\sigma_{m} \approx 2 \pi R^{2}$ when $k_{m} R \rightarrow \infty$ to obtain

$$
\operatorname{var}\left\{u_{m}(Z)\right\}=\left|u_{m o}\right|^{2} e^{-2 \pi \int_{0}^{Z} N(z) R^{2} d z}\left[e^{\pi \int_{0}^{Z} N(z) R^{2} d z}-1\right]
$$

When the inclusions are all uniform with respect to size, elastic parameters, and density, the result for high frequencies reduces to the simple form

$$
\operatorname{var}\left\{u_{m}(Z)\right\}=\left|u_{m o}\right|^{2} e^{-2 \pi N R^{2} Z}\left[e^{\pi N R^{2} Z}-1\right]
$$

This result says that for scatterers large compared to the wavelength of the incident wave, the fluctuations in the scattered field are independent of frequency and proportional to the density of scatterers $N$ and the squared radius of the inclusions.

Consider again the general expression for the variance given in equation (32). It is straightforward to show in both of the limit cases $\omega \rightarrow 0$ and $\omega \rightarrow \infty$ that

$$
2 \pi\left|A_{m}(z)\right|^{2} G_{m}<\sigma_{m}(z)
$$

Although an analytical demonstration has not yet been found, numerical calculations indicate that this result holds for all frequencies. Assuming this to be true in general, it follows that $\operatorname{var}\left\{u_{m}(Z)\right\} \rightarrow 0$ as $Z \rightarrow \infty$. From equation (32) it is obvious that $\operatorname{var}\left\{u_{m}(Z)\right\} \rightarrow 0$ as $Z \rightarrow 0$. Thus the variance must have a maximum at some intermediate value $Z=\hat{Z}$. The condition for this maximum is

$$
2 \pi \int_{0}^{\hat{Z}} N(z)\left|A_{m}(z)\right|^{2} G_{m} d z=\ln \left[\frac{\sigma_{m}(\hat{Z})}{\sigma_{m}(\hat{Z})-2 \pi\left|A_{m}(\hat{Z})\right|^{2} G_{m}}\right]
$$


Introducing the concentration $c(z)=\frac{4 \pi}{3} R^{3} N(z)$ as the fraction of the volume occupied by inclusions and assuming that it and the inclusion properties are constant at low frequencies leads to

$$
c \frac{\hat{Z}}{R}=\frac{8 k_{m}^{2} R^{4}}{27 \pi\left|A_{m}\right|^{2}} \ln \left[\frac{\sigma_{m}}{\sigma_{m}-2 \pi\left|A_{m}\right|^{2} G_{m}}\right]
$$

\section{Attenuation for small scatterers}

In the low frequency range where the inclusions are small compared to a wavelength (Rayleigh scattering), the scattering coefficients have fairly simple forms for the case of a homogeneous spherical

inclusion. From Korneev and Johnson (1996) we have the (un-normalized) scattering cross sections

$$
\begin{aligned}
& \sigma_{p}=\frac{4 \pi}{9} k_{p}^{4} R^{6}\left\{\left(\frac{\frac{3}{2}\left(\lambda_{1}-\lambda_{2}\right)+\mu_{1}-\mu_{2}}{\frac{3}{2} \lambda_{1}+\mu_{1}+2 \mu_{2}}\right)^{2}+\frac{1}{3}\left(\frac{\rho_{1}}{\rho_{2}}-1\right)^{2}\left(1+\frac{2}{\gamma^{2}}\right)\right. \\
& \left.+\frac{8}{45}\left(\frac{\mu_{1}}{\mu_{2}}-1\right)^{2} \frac{\gamma^{2}}{D^{2}}\left(2 \gamma^{2}+\frac{3}{\gamma^{2}}\right)\right\} \\
& \sigma_{s}=\frac{4 \pi}{27} k_{s}^{4} R^{6}\left\{\left(\frac{\rho_{1}}{\rho_{2}}-1\right)^{2}\left(\gamma^{3}+2\right)+\frac{2}{5}\left(\frac{\mu_{1}}{\mu_{2}}-1\right)^{2} \frac{1}{D^{2}}\left(2 \gamma^{5}+3\right)\right\}
\end{aligned}
$$

Also,

$$
\operatorname{Re}\left\{A_{m}\right\}=-\frac{2}{3} k_{m}^{2} R^{3} \Delta_{m}
$$

where

$$
\Delta_{p}=\frac{1}{2}\left\{\frac{\frac{3}{2}\left(\lambda_{1}-\lambda_{2}\right)+\mu_{1}-\mu_{2}}{\frac{3}{2} \lambda_{1}+\mu_{1}+2 \mu_{2}}-\left(\frac{\rho_{1}}{\rho_{2}}-1\right)+\frac{4}{3}\left(\frac{\mu_{1}}{\mu_{2}}-1\right) \frac{\gamma^{2}}{D}\right\}
$$

and

$$
\Delta_{s}=\frac{1}{2}\left\{\left(\frac{\mu_{1}}{\mu_{2}}-1\right) \frac{1}{D}-\left(\frac{\rho_{1}}{\rho_{2}}-1\right)\right\}
$$

$D$ is defined in Appendix A.

Using equation (42) and substituting the concentration that was previously defined, the time shift of transmitted waves from equation (18) becomes

$$
\Delta t_{m}(Z)=\frac{1}{v_{m}} \int_{0}^{Z} c(z) \Delta_{m}(z) d z
$$


The time shift parameter of equation (20) is now

$$
\overline{\Delta_{m}}(Z)=\frac{1}{Z} \int_{0}^{Z} c(z) \Delta_{m}(z) d z
$$

which is independent of frequency. Thus at low frequencies there is a constant time shift and a nondispersive effective velocity.

For the low-contrast case where

$$
\frac{|\delta \lambda|}{\lambda}=\frac{\left|\lambda_{1}-\lambda_{2}\right|}{\lambda} \ll 1, \frac{|\delta \mu|}{\mu}=\frac{\left|\mu_{1}-\mu_{2}\right|}{\mu} \ll 1, \frac{|\delta \rho|}{\rho}=\frac{\left|\rho_{1}-\rho_{2}\right|}{\rho} \ll 1
$$

the expressions in equations (41) and (43) simplify to

$$
\begin{aligned}
& \sigma_{p}=\frac{4 \pi}{9} k_{p}^{4} R^{6}\left\{\left(\frac{\delta \lambda+\frac{2}{3} \delta \mu}{\lambda+2 \mu}\right)^{2}+\frac{1}{3}\left(\frac{\delta \rho}{\rho}\right)^{2}\left(1+\frac{2}{\gamma^{2}}\right)+\frac{8}{45}\left(\frac{\delta \mu}{\mu}\right)^{2} \gamma^{2}\left(2 \gamma^{2}+\frac{3}{\gamma^{2}}\right)\right\} \\
& \sigma_{s}=\frac{4 \pi}{27} k_{s}^{4} R^{6}\left\{\left(\frac{\delta \rho}{\rho}\right)^{2}\left(2+\gamma^{2}\right)+\frac{2}{5}\left(\frac{\delta \mu}{\mu}\right)^{2}\left(2 \gamma^{5}+3\right)\right\} \\
& \Delta_{p}=\frac{1}{2}\left\{\frac{\delta \lambda+2 \delta \mu)}{\lambda+2 \mu}-\frac{\delta \rho}{\rho}\right\}=\frac{\delta v_{p}}{v_{p}} \\
& \Delta_{s}=\frac{1}{2}\left\{\frac{\delta \mu}{\mu}-\frac{\delta \rho}{\rho}\right\}=\frac{\delta v_{s}}{v_{s}}
\end{aligned}
$$

The time shift parameter of equation (44) then has the simple form

$$
\overline{\Delta_{m}}(Z)=\frac{1}{Z} \int_{0}^{Z} c(z) \frac{\delta v_{m}(z)}{v_{m}} d z
$$

so that

$$
\Delta t_{m}=\int_{0}^{Z} c(z) \frac{\delta v_{m}(z)}{v_{m}^{2}} d z
$$

Note that when $c(z)$ and $\delta v_{m}(z)$ are constant, equation (21) can be put in the form

$$
\frac{v_{m}^{e f}-v_{m}}{v_{m}^{e f}} \equiv \frac{\delta v_{m}^{e f}}{v_{m}^{e f}}=c \frac{\delta v_{m}}{v_{m}}
$$

This is a particularly simple relationship between the relative change in the effective velocity and the relative change in the inclusion velocity. 
It is instructive to compare these low-frequency results with the effective media results of Kuster and Toksöz (1974). They used a low-frequency single-scattering approach to derive relationships between effective elastic moduli and the individual moduli of the composite material components. Their results can be used to estimate the velocity of $\mathrm{P}$ and $\mathrm{S}$ waves, but not the attenuation. In terms of the notation used in this paper, their results are obtained by solving the following system of equations

$$
\begin{aligned}
& \frac{K_{e f}-K_{2}}{3 K_{e f}+4 \mu_{2}}=c \frac{K_{1}-K_{2}}{3 K_{1}+4 \mu_{2}} \\
& \frac{\mu_{e f}-\mu_{2}}{6 \mu_{e f}\left(K_{2}+2 \mu_{2}\right)+\mu_{2}\left(9 K_{2}+8 \mu_{2}\right)}=c \frac{\mu_{1}-\mu_{2}}{6 \mu_{1}\left(K_{2}+2 \mu_{2}\right)+\mu_{2}\left(9 K_{2}+8 \mu_{2}\right)} \\
& \rho_{e f}-\rho_{2}=c\left(\rho_{1}-\rho_{2}\right)
\end{aligned}
$$

where the following bulk moduli have been introduced

$$
K_{1}=\lambda_{1}+\frac{2}{3} \mu_{1}, K_{2}=\lambda_{2}+\frac{2}{3} \mu_{2}, K_{e f}=\lambda_{e f}+\frac{2}{3} \mu_{e f}
$$

We have performed a numerical comparison between the low-frequency formulas for velocity derived in this paper and those obtained with the approach of Kuster and Toksöz (1974). The matrix medium was chosen with properties:

$$
v_{p 2}=5.3 \mathrm{~km} / \mathrm{s}, \quad v_{s 2}=3.2 \mathrm{~km} / \mathrm{s}, \rho_{2}=2.65 \mathrm{gm} / \mathrm{cm}^{3}
$$

In Figure $2 a$ the effective velocities $v_{p}^{e f}$ and $v_{s}^{\text {ef }}$ calculated by both methods are plotted as functions of concentration $c$ for low-velocity scatterers with properties:

$$
v_{p 1}=3.0 \mathrm{~km} / \mathrm{s}, v_{s 1}=2.0 \mathrm{~km} / \mathrm{s}, \rho_{1}=2.6 \mathrm{gm} / \mathrm{cm}^{3}
$$

Figure $2 \mathrm{~b}$ shows a similar comparison for high-velocity scatterers with properties:

$$
v_{p 1}=7.0 \mathrm{~km} / \mathrm{s}, \quad v_{s 1}=4.0 \mathrm{~km} / \mathrm{s}, \rho_{1}=3.0 \mathrm{gm} / \mathrm{cm}^{3}
$$


The two methods give almost the same results for both low-velocity and high-velocity inclusions for the low concentrations where the theories are valid. These results demonstrate that at low frequencies the results of this paper are entirely consistent with effective media expressions, such as those of Kuster and Toksöz (1974). It is worth noting that these results should only be used for concentrations $c<0.5$, as the meanings of "matrix" and "inclusion" are reversed for larger values.

\section{Numerical modeling of scattering}

The effect of scattering on propagating plane elastic waves was simulated by using the exact scattering solution for a single elastic sphere (Korneev and Johnson, 1993a,1996). The design of the experiment is shown in Figure 1. A thin slab of a scattering medium having thickness $\Delta z$ was simulated by distributing a large number of spherical inclusions having the same radius $R$ and with random spacing so that the $z$ coordinates of all the centers of the spheres were in the interval $(-\Delta z, 0)$. Plane elastic $\mathrm{P}$ and $\mathrm{S}$ wave pulses containing a broad range of frequencies were propagated in the positive $\mathrm{z}$ direction. Offset a distance $h$ from the scattering region, were a set of $K$ receivers having a separation interval of $\Delta x$. The lateral size of the box, $20 \mathrm{~km} \times 20 \mathrm{~km}$, was taken large in comparison to $h$ and $\Delta x$ in order to achieve the effect of a layer having infinite extent. The concentration was taken to be $c=10 \%$, which required a rather large number of scatterers $N=5300$. The other parameters used were $h=4 \mathrm{~km}, R=0.1 \mathrm{~km}, \Delta z=0.5 \mathrm{~km}$ and $\Delta x=0.5 \mathrm{~km}$. The material properties of the background medium were

$$
v_{p 2}=5.3 \mathrm{~km} / \mathrm{s}, v_{s 2}=3.2 \mathrm{~km} / \mathrm{s}, \rho_{2}=2.65 \mathrm{gm} / \mathrm{cm}^{3}
$$

and for the scatterers they were

$$
v_{p 1}=3.0 \mathrm{~km} / \mathrm{s}, \quad v_{s 1}=2.0 \mathrm{~km} / \mathrm{s}, \rho_{1}=2.6 \mathrm{gm} / \mathrm{cm}^{3}
$$


The calculated transmitted seismograms at 20 different values of $x$ for both an incident $S$ wave and an incident $\mathrm{P}$ wave are shown in Figure 3. In both cases there is a fairly coherent direct wave followed by a long coda of incoherent scattered waves. Note that only the principal component of the incident wave is shown, which is the $x$ component for an incident $\mathrm{S}$ wave and the $z$ component for an incident $\mathrm{P}$ wave. This explains why there is not a significant amount of scattered energy preceding the coherent transmitted S wave in this figure, as this energy appears on the $z$ component. There is also some low level noise present on the traces due to the wrap-around associated with the finite time window used in the numerical Fourier transform.

The Fourier transforms of the seismograms shown in Figure 3 were calculated and then the relationships of equations (12) and (13) used to estimate the attenuation parameter $\Delta \bar{q}_{m}(z+\Delta z)$ from the modulus and the phase shift parameter $\Delta \bar{\phi}_{m}(z+\Delta z)$ from the phase. The results are shown as dotted lines in Figure 4 (incident $\mathrm{S}$ wave) and in Figure 5 (incident $\mathrm{P}$ wave). In each case the calculations were performed on a single seismogram (upper panels in the figures) and for the average of all 20 seismograms (lower panels in the figures). Note that the phase is ambiguous by multiples of $2 \pi$ and this ambiguity can sometimes be removed by searching for discontinuities. This unwrapping of the phase was attempted for the phase calculated from the averaged seismogram, but not for that of the single seismogram where the high degree of randomness in the data did not permit a stable result. Also shown in Figures 4 and 5 are the analytical estimates for the attenuation and phase calculated from equation (8) (heavy solid lines) and the analytical estimates for the fluctuations calculated from equation (24) (light solid lines shown as plus and minus one standard deviation). For the comparison with the average seismograms it was assumed that the fluctuations accumulated randomly so that the variance of the average decreased as $K^{-1}$, where $K=20$ was the number of seismograms that were averaged. 
The results of Figures 4 and 5 form the basis for several general observations. First, the analytical estimates are in reasonable agreement with the numerical results for both the mean and variance. For a single seismogram the statistical uncertainty is so large that only general trends can be identified, but in the case of the averaged seismograms a much more quantitative evaluation of the agreement can be performed. The agreement is slightly better for the attenuation than for the phase, which contains the additional complication of phase unwrapping. An associated observation is that, due to the statistical fluctuations, it may be impractical in many situations to reliably estimate attenuation and phase from a single seismogram, and the advantages of using spatially averaged data are significant. A second general observation is that the results exhibit relatively simple behavior at low frequencies where the wave parameter is less than about 2 . In this range the numerical and analytical results are in good agreement (even for a single seismogram) and the phase shows a linear dependence upon frequency. The third general observation concerns the behavior at high frequencies. Although there are both long and short wavelength oscillations in the results that are associated with the dimensions and properties of the scatterers, the trend in the attenuation is a constant non-zero value and the trend in the phase is a constant value of about zero.

In the discussion following equation (32) it was pointed out that, due to the competing effects of conversion of energy from the coherent field and the resultant decay of the coherent field, the amplitude of the fluctuations in the transmitted field will have a maximum at a particular propagation distance. Note that an expression for the maximum is given in equation (40), but this is not a particularly simple function of either frequency or wave type. This phenomenon is shown for various values of frequency in Figure 6, where it is clear that the fluctuations rise from a value of zero at small distances and decay to zero again at large distances, which requires that there be a maximum at some intermediate distance. It appears that the distance to the maximum decreases as 
the frequency increases. It also appears in this figure that the distance to the maximum is greater for $S$ waves than $P$ waves at low frequencies, but the situation is reversed at high frequencies. These tendencies are shown more clearly in Figure 7, which graphs the position of the maximum as a function of frequency. Here it is clear that the distance to the maximum decreases rapidly at low frequencies, but then stabilizes at a fairly constant value at high frequencies. Also, while the distance to the maximum is greater for $S$ waves than $P$ waves at low frequencies, this relationship begins to alternate with increasing frequency and both types of waves show similar behavior at the highest frequencies.

Equation (21) shows how the phase shift of the coherent transmitted wave can be converted to an effective velocity by interpreting the phase as a time shift. Applying this result to the average of the seismograms in Figure 3 (thus using the mean phase in Figures $4 \mathrm{~d}$ and $5 \mathrm{~d}$ ) results in the dispersion curves shown in Figure 8. In this example of low velocity inclusions the velocities at low frequencies (Rayleigh scattering regime) are less than the background values and thus represent effective media values, as predicted by the results of Figure 2a. At high frequencies (ray theory regime) the velocities approach those of the background, as expected. At intermediate frequencies, there is a more complex dependence upon frequency that includes both minima and maxima in the dispersion curves, with these features associated with the minima and maxima of the phase curves in Figures $4 \mathrm{~d}$ and $5 \mathrm{~d}$. Note that these minima and maxima do not have to be at the same frequency for $\mathrm{P}$ and $\mathrm{S}$ waves, so the ratio of these velocities can exhibit an even more complicated behavior.

\section{Discussion and conclusions}

The analytical results of this paper provide a method of estimating the effects of scattering upon a plane wave propagating through a layer of randomly distributed spherical inclusions. Formulas 
have been obtained for both the average field and the statistical fluctuations about this average. The general results can be used for inclusions of arbitrary size and contrast and for all frequencies, but approximations for small inclusions or low contrast inclusions have also been included. These analytical results have been validated by comparing them with effective media estimates at low frequencies and with numerical simulations over the entire frequency range. In both cases the agreement is satisfactory, which suggests that appropriate approximations were used in evaluating the various integrals encountered in the theoretical development.

The results displayed in Figures 4 and 5 show that the relatively simple expressions of equations (14) and (15) do an acceptable job over a broad frequency range of describing phase and attenuation effects upon a wave propagating through a region containing scatterers. The fluctuations calculated with equation (32) also serve as adequate bounds on the statistical uncertainty of the mean field. What is also clear in Figures 4 and 5 (panels a and b) is that, because of the random fluctuations, any attempt to reliably estimate the characteristics of the scattering on the basis of a single seismogram may be a difficult task. Only the phase shift at low frequencies shows a reasonable approximation to the mean field. Note that non-physical negative values of attenuation are common in the results for a single seismogram. This means that in most cases some sort of spatial averaging of the observational data will be necessary before stable values of the mean phase and attenuation can be estimated.

Assuming that an averaging process has been applied which has reduced the fluctuations to a acceptable fraction of the mean value, it is of interest to consider how data such as that shown in Figures 4 and 5 can be interpreted in terms of material properties of the scattering medium. Measurements at high frequencies of velocity (or phase) are only dependent upon the background medium and thus can be used to estimate $v_{p 2}, v_{s 2}$, and $\rho_{2}$. Measurements of velocity at low frequencies can be combined with equations (21), (43), and (44) to provide constraints on the 
properties of the inclusions $v_{p 1}, v_{s 2}, \rho_{2}$ and the concentration $c$. For the low-contrast case the even simpler result of equation (48) can be used. The first peak in the phase curve is a well defined feature and constraints on the material properties can be obtained by fitting the analytical results to the data. For instance, in the low-contrast case it can be shown that the position of this first peak is given by

$$
\omega_{\text {peak }} \approx 2.8 \frac{v_{m 2}}{2 R}\left(\frac{v_{m 2}}{v_{m 1}}-1\right)^{\frac{1}{2}}
$$

Similar constraints are provided by measurements of attenuation. Using equation (16), and assuming a uniform distribution of the concentration and type of inclusions, we have

$$
\bar{q}_{m}(Z)=\frac{3 c Z}{8 \pi R^{3}} \sigma_{m}
$$

At low frequencies (Rayleigh regime) where the attenuation is rapidly increasing, the proportionality of $\sigma_{m} \sim \omega^{4} R^{6}$ can be used to obtain

$$
\bar{q}_{m}(Z) \sim c R^{3} Z \omega^{4}
$$

Fitting this expression (including the appropriate proportionality factors from equation 41 ) to the attenuation places constraints on $c$ and $R$. At high frequencies $\left(k_{m} R \gg 1\right)$ where the attenuation is approximately constant the limiting value of $\sigma_{m}=2 \pi R^{2}$ leads to

$$
\bar{q}_{m}(Z)=\frac{3 c Z}{4 R}
$$

This is second constraint on $c$ and $R$ provided by the attenuation data, which means that independent estimates of these two parameters are possible. Note that in the limits of low and high frequencies the attenuation data are independent of the velocities and densities. Also note that the phase data are mainly sensitive to the material properties, while the attenuation data are mainly sensitive to the geometry of the inclusions. 
Some information about the properties of the scattering medium can also be extracted from the level of the fluctuations. At high frequencies this level becomes independent of frequency. Noting that the mean value of the phase goes to zero in this range, one can start with equation (34) and use the same arguments that led to equation (38) to obtain

$$
\operatorname{var}\left\{\phi_{m}(Z)\right\}=\frac{\pi N R^{2} Z}{2}=\frac{3 c Z}{8 R}
$$

This provides another constraint on $\mathrm{c}$ and $\mathrm{R}$.

In this paper the attenuation has been described with the non-dimensional attenuation $\bar{q}_{m}$ or the attenuation parameter $\alpha_{m}$. Another common method of defining attenuation is in terms of the quality factor $Q$. The relationships between the various definitions are

$$
Q_{m}^{-1}=\frac{2 v_{m}}{\omega Z} q_{m}=\frac{v_{m}}{\omega} \alpha_{m}=\frac{v_{m}}{\omega} N \sigma_{m}=\frac{3 c v_{m}}{4 \pi \omega R^{3}} \sigma_{m}
$$

Using the same limits discussed above, at low frequencies we have

$$
Q_{m}^{-1} \sim c R^{3} \omega^{3}
$$

and at high frequencies

$$
Q_{m}^{-1}=\frac{3 c v_{m}}{2 \omega R}
$$

The results of this paper have been demonstrated with calculations and examples that assumed inclusions having the same type and size, but the results are much more general than this. For instance, in the basic results of equations (14), (15), and (32) the critical elements are integrals involving expressions of the form $N(z) F\left\{A_{m}(z)\right\}$, where $N(z)$ is the density of scatterers having the forward scattering coefficients $A_{m}(z)$ and $F$ is some function of $A_{m}$. This is easily generalized to the situation where there are $J$ different types of scatterers, each having its own distribution in density $N^{(j)}(z)$ and scattering coefficients $A_{m}^{(j)}(z)$. Then the basic results involve integrals of the 
form

$$
\int_{0}^{Z} N(z) F\left\{A_{m}(z)\right\} d z=\sum_{j=1}^{J} \int_{0}^{Z} N^{(j)}(z) F\left\{A_{m}^{(j)}(z)\right\} d z
$$

This allows calculations to be made for rather arbitrary distributions of inclusions, with the only restrictions being that the distributions only be a function of $z$ and that the total concentration be small.

The results presented in this paper are only valid for small values of the concentration $c$. This restriction can be removed for the case of the average field, and Kaelin and Johnson (1998) develop a self-consistent version of the results in this paper for the coherent wave. What results is an implicit expression for the attenuation and phase, which means that an optimization problem has to be solved at each frequency. An equivalent treatment has not yet been developed for the fluctuations in the transmitted wave.

\section{Acknowledgments}

This work was supported by the Assistant Secretary for Fossil Energy, Office of Oil, Gas and Shale Technologies, Federal Energy Technology Center, of the U.S. Department of Energy under Contract No. DE-AC03-76SF00098. Support was also provided by the Defense Special Weapons Agency under Grant No. DSWA-01-97-1-0026. All computations were carried out at the Center for Computational Seismology, which is supported by the DOE/BES Geoscience Program at the Ernest Orlando Lawrence Berkeley National Laboratory.

\section{Appendix A}

In evaluating fluctuations in the scattered field it is necessary to take into account the angular dependence of the scattering coefficients. Expressions for the angular scattering coefficients $\mathbf{A}_{m p}(\theta, \phi)$ 
and $\mathbf{A}_{m s}(\theta, \phi)$ can be found in Korneev and Johnson (1996) for the case where the inclusion is a homogeneous sphere of radius $R$. Here we will need approximate results for the Mie scattering regime $k_{m} R \approx 1$. For the forward scattering direction and an incident $\mathrm{P}$ wave this is

$$
\left[\mathbf{A}_{p p}(\theta, \phi)\right]_{z} \approx 3 A_{p}(0) \frac{j_{1}\left(\beta_{p}\right)}{\beta_{p}} \cos \theta
$$

where

$$
A_{p}(0)=\frac{k_{p}^{2} R^{2}}{3}\left\{-\frac{1}{2} \frac{\frac{3}{2}\left(\lambda_{1}-\lambda_{2}\right)+\mu_{1}-\mu_{2}}{\frac{1}{2}\left(\frac{3}{2} \lambda_{1}+\mu_{1}\right)+\mu_{2}}+\left(\frac{\rho_{1}}{\rho_{2}}-1\right)-\frac{4}{3}\left(\frac{\mu_{1}}{\mu_{2}}-1\right) \frac{\gamma^{2}}{D}\right\}
$$

Here $j_{1}$ is the spherical Bessel function of the first order, and

$$
\begin{aligned}
& \beta_{p}=2 k_{p} R \sin \frac{\theta}{2} \\
& D=1+\frac{2}{15}\left(\frac{\mu_{1}}{\mu_{2}}-1\right)\left(3+2 \gamma^{2}\right)
\end{aligned}
$$

For an incident $\mathrm{S}$ wave the corresponding result is

$$
\left[\mathbf{A}_{s s}(\theta, \phi)\right]_{x} \approx 3 A_{s}(0) \frac{j_{1}\left(\beta_{s}\right)}{\beta_{s}} \cos \theta
$$

where

$$
A_{s}(0)=\frac{k_{s}^{2} R^{2}}{3}\left\{\left(\frac{\rho_{1}}{\rho_{2}}-1\right)-\frac{1}{D}\left(\frac{\mu_{1}}{\mu_{2}}-1\right)\right\}
$$

and

$$
\beta_{s}=2 k_{s} R \sin \frac{\theta}{2}
$$

At high frequencies $k_{m} R \gg 1$ it is more valid to use the approximation obtained by Dubrovsky and Morochnik (1983) for low-contrast spherical inclusions.

$$
\left[\mathbf{A}_{m m}(\theta, \phi)\right]_{m}=-i k_{m} R^{2} \sqrt{\frac{\theta}{\sin \theta}}\left(\frac{J_{1}\left(k_{m} R \theta\right)}{k_{m} R \theta}-i \frac{\delta_{m}}{\eta_{m}^{2}} \exp \left(-i \eta_{m}\right)\right)
$$

where $J_{1}$ is the cylindrical Bessel function and

$$
\eta_{m}=k_{m} R\left(4\left(\frac{v_{m}^{(2)}}{v_{m}^{(1)}}-1\right)^{2}+\theta^{2}\right)^{\frac{1}{2}}, \quad \delta_{m}=2 k_{m} R\left(\frac{v_{m}^{(2)}}{v_{m}^{(1)}}-1\right)
$$


Because the forward scattering direction where $\theta$ is small is of primary interest, equation (A-3) can be approximated so that it is normalized for the arbitrary-contrast high-frequency case and then it becomes

$$
\left[\mathrm{A}_{m m}(\theta, \phi)\right]_{c_{m}}=2 A_{m}(z) \sqrt{\frac{\theta}{\sin \theta}} \frac{J_{1}\left(k_{m} R \theta\right)}{k_{m} R \theta}
$$

With the above results it is now possible to calculate the squared modulus of the change in the incident wave, which is useful when considering random fluctuations in the scattered field. For the Mie case this is

$$
\left|\Delta u_{m}^{(1)}\right|^{2}=9\left|u_{m o}\right|^{2}\left|A_{m}(0)\right|^{2} \frac{\cos ^{2} \theta}{r^{2}} \frac{j_{1}^{2}\left(\beta_{m}\right)}{\beta_{m}^{2}}
$$

For the high frequency case it is

$$
\left|\Delta u_{m}^{(1)}\right|^{2}=4\left|u_{m o}\right|^{2}\left|A_{m}(0)\right|^{2} \frac{1}{r^{2}} \frac{J_{l}^{2}\left(k_{m} R \theta\right)}{k_{m}^{2} R^{2} \theta \sin \theta}
$$

It will be shown later that for the purposes of this paper the difference between these last two equations is insignificant.

\section{Appendix B}

To evaluate the integral associated with the fluctuations in the transmitted field

$$
I=\int_{\infty}^{\infty} \int_{\infty}^{\infty}\left|\left[\mathbf{A}_{m m}(\theta, \phi)\right]_{c_{m}}\right|^{2} \frac{d x d y}{r^{2}}
$$

it is necessary to use more accurate expressions for the scattering function $\left[\mathbf{A}_{m m}(\theta, \phi)\right]_{c_{m}}$ than for the case of the average field (equation 7). We no longer can take advantage of an oscillating factor which tended to concentrate the contribution in the vicinity of $\theta \approx 0$, but must now integrate over a wider aperture, which requires a more accurate specification of the angular dependence of the scattering function. Therefore we use a scattering function in the form

$$
\left[\mathbf{A}_{m m}(\theta, \phi)\right]_{c_{m}}=3 \frac{j_{1}\left(\beta_{m}\right)}{\beta_{m}} \frac{\left|A_{m}(z)\right|}{\left|\mathbf{R}_{m}(0,0)\right|}\left[\mathbf{R}_{m m}(\theta, \phi)\right]_{c_{m}}, \quad c_{m}= \begin{cases}z, & m=p \\ x, & m=s\end{cases}
$$


where $\mathbf{R}_{m m}(\theta, \phi)$ is the Rayleigh solution for the sphere of an arbitrary contrast taken from Korneev and Johnson (1996). Introduction of the ratio $\frac{\left|A_{m}(z)\right|}{\left|\mathrm{R}_{m}(0,0)\right|}$, with function $A_{m}(z)$ from equation (9) allows the applicability of equation (B-2) to be extended to higher frequencies while still taking advantage of the main contribution of $\left[\mathbf{A}_{m m}(\theta, \phi)\right]_{c_{m}}$ in equation (B-1) for small angles.

For an incident $\mathrm{P}$ wave we have

$$
\frac{\left[\mathbf{R}_{p p}(\theta, \phi)\right]_{z}}{\left|\mathbf{R}_{p}(0,0)\right|}=\cos \theta\left(p_{1}+p_{2} \cos \theta+p_{3} \cos ^{2} \theta\right)
$$

where we have introduced the notation

$$
\begin{aligned}
& p_{1}=\frac{a_{1}}{a_{0}}, \quad p_{2}=\frac{a_{2}}{a_{0}}, \quad p_{3}=\frac{a_{3}}{a_{0}} \\
& a_{0}=a_{1}+a_{2}+a_{3} \\
& a_{1}=-\frac{1}{2} \frac{\frac{3}{2}\left(\lambda_{1}-\lambda_{2}\right)+\mu_{1}-\mu_{2}}{\frac{3}{2} \lambda_{1}+\mu_{1}+2 \mu_{2}}+\frac{2}{3}\left(\frac{\mu_{1}}{\mu_{2}}-1\right) \frac{\gamma^{2}}{D} \\
& a_{2}=\left(\frac{\rho_{1}}{\rho_{2}}-1\right) \\
& a_{3}=-2\left(\frac{\mu_{1}}{\mu_{2}}-1\right) \frac{\gamma^{2}}{D}
\end{aligned}
$$

Substituting equation (B-3) in equation (B-1) results in

$$
\overline{\left|U_{p}\right|^{2}}=\int_{\infty}^{\infty} \int_{\infty}^{\infty}\left|\left[\mathbf{A}_{p p}(\theta, \phi)\right]_{z}\right|^{2} \frac{d x d y}{r^{2}}=2 \pi\left|A_{p}(z)\right|^{2} G_{p}
$$

where

$$
G_{p}=\sum_{k=2}^{6} g_{k}\left(w_{p}\right) P_{k}, \quad w_{p}=2 \frac{\omega R}{v_{p 2}}
$$

and the coefficients $P_{k}$ have the simple forms

$$
\begin{gathered}
P_{2}=p_{1}^{2}, \quad P_{3}=2 p_{1} p_{2}, \quad P_{4}=2 p_{1} p_{3}+p_{2}^{2} \\
P_{5}=2 p_{2} p_{3}, \quad P_{6}=p_{3}^{2}
\end{gathered}
$$


The functions $g_{k}(w)$ are integrals of the form

$$
g_{k}\left(w_{m}\right)=9 \int_{0}^{\infty} \frac{j_{1}^{2}\left(w_{m} \sin \frac{\theta}{2}\right)}{\left(w_{m} \sin \frac{\theta}{2}\right)^{2}} \cos ^{k} \theta \frac{\rho d \rho}{r^{2}}=\frac{36}{w_{m}^{2}} \int_{0}^{\frac{w_{m}}{\sqrt{2}}} \frac{j_{1}^{2}(t)}{t}\left(1-\frac{2 t^{2}}{w_{m}^{2}}\right)^{k+1} d t
$$

These integrals can be analytically evaluated for low values of $\mathrm{k}$. For instance, when $k=0$ we have

$$
g_{0}=\frac{9}{2 d^{2}}\left(1+\frac{1}{d^{2}}-\frac{\sin 2 d}{d^{3}}+\frac{\sin ^{2} d}{d^{4}}-\frac{4}{d^{2}} \int_{0}^{d} \frac{\sin ^{2} t}{t} d t\right), \quad d=w_{m} / \sqrt{2}
$$

As $k$ increases the expressions for $g_{k}(w)$ become increasingly bulky and contain special functions similar to the $g_{0}$ case. An examination of these integral expressions revealed that, for the purposes of this paper, the exact expressions were not necessary and the following approximate expressions were sufficiently accurate

$$
\tilde{g}_{k}\left(w_{m}\right)=\frac{1}{4(k+2)\left(1+\frac{w_{m}^{2}}{36}(k+2)\right)}, \quad k=0,1,2, \ldots
$$

A demonstration of this fact is given in Figure B-1, where the exact (equation B-6) and approximate (equation B-8) expressions are compared for $k=0, \ldots 6$. Note that for large arguments (high frequencies) all $g_{k}$ approach the same asymptotic value

$$
g_{k}\left(w_{m}\right) \approx \frac{9}{w_{m}^{2}}
$$

In the case of an incident $S$ wave we have

$$
\frac{\left[\mathbf{R}_{s s}(\theta, \phi)\right]_{x}}{\left|\mathbf{R}_{s}(0,0)\right|}=S_{1}\left(\sin ^{2} \phi+\cos ^{2} \phi \cos ^{2} \theta\right)+S_{2}\left(\cos ^{2} \phi-\sin ^{2} \phi-2 \cos ^{2} \phi \cos ^{2} \theta\right) \cos \theta
$$

where

$$
\begin{aligned}
& s_{1}=\frac{b_{1}}{b_{0}}, \quad s_{2}=\frac{b_{2}}{b_{0}}, \\
& b_{0}=b_{1}-b_{2} \\
& b_{1}=\left(\frac{\rho_{1}}{\rho_{2}}-1\right) \\
& b_{2}=\left(\frac{\mu_{1}}{\mu_{2}}-1\right) \frac{1}{D}
\end{aligned}
$$


Corresponding to equation (B-4), we have for $S$ waves

$$
\overline{\left|U_{s}\right|^{2}}=\int_{\infty}^{\infty} \int_{\infty}^{\infty}\left|\left[\mathbf{A}_{s s}(\theta, \phi)\right]_{x}\right|^{2} \frac{d x d y}{r^{2}}=2 \pi\left|A_{s}(z)\right|^{2} G_{s}
$$

where

$$
G_{s}=\sum_{k=0}^{6} g_{k}\left(w_{s}\right) S_{k}, \quad w_{s}=2 \frac{\omega R}{V_{s 2}}
$$

and the $g_{k}$ functions are the same as those defined in equation (B-6). The coefficients $S_{k}$ are given by

$$
\begin{aligned}
& S_{0}=\frac{3}{8} s_{1}^{2}, \quad S_{1}=-\frac{1}{2} s_{1} s_{2}, \quad S_{2}=\frac{1}{4}\left(s_{1}^{2}+2 s_{2}^{2}\right), \quad S_{3}=0 \\
& S_{4}=\frac{3}{8} s_{1}^{2}-s_{2}^{2}, \quad S_{5}=-\frac{3}{4} s_{1} s_{2}, \quad S_{6}=\frac{3}{2} s_{2}^{2},
\end{aligned}
$$

It is easy to show that at low frequencies the functions

$$
G_{m}(w)=G_{m}^{(0)}
$$

do not depend on frequency and satisfy the inequalities

$$
\left|G_{m}^{(0)}\right|<1
$$

whereas at high frequencies they approach

$$
G_{m}(\omega)=\left(\frac{3 v_{m}}{2 \omega R}\right)^{2}
$$

\section{Appendix C}

The body of this paper contains expressions for the mean field and the mean squared field as it propagates through a layer containing randomly distributed scatterers. It is desirable to have similar results for the phase shift and attenuation of the propagating wave. Starting with equation (5), the primary component of the total field after interaction with a single scatterer is

$$
\mathbf{u} \cdot \hat{\mathbf{u}}_{m}=u_{m o}+\Delta u_{m}^{(1)}=u_{m o}\left[1+\left[\mathbf{A}_{m m}(\theta, \phi)\right]_{c_{m}} \frac{e^{-i k_{m}(r-z)}}{r}\right]
$$


In terms of increments in phase and attenuation this can also be represented as

$$
\mathbf{u} \cdot \hat{\mathbf{u}}_{m}=u_{m o} e^{\left(i \Delta \phi_{m}-\Delta q_{m}\right)}
$$

Assuming the change in phase and attenuation are small, this is

$$
\mathbf{u} \cdot \hat{\mathbf{u}}_{m} \approx u_{m o}\left[1+i \Delta \phi_{m}-\Delta q_{m}\right]
$$

Comparing this with equation (C-1) yields

$$
i \Delta \phi_{m}-\Delta q_{m}=\left[\mathbf{A}_{m m}(\theta, \phi)\right]_{c_{m}} \frac{e^{-i k_{m}(r-z)}}{r}
$$

or, equivalently,

$$
\begin{aligned}
& \Delta \phi_{m}=\frac{1}{r} \operatorname{Im}\left\{\left[\mathrm{A}_{m m}(\theta, \phi)\right]_{c_{m}} e^{-i k_{m}(r-z)}\right\} \\
& \Delta q_{m}=-\frac{1}{r} \operatorname{Re}\left\{\left[\mathbf{A}_{m m}(\theta, \phi)\right]_{c_{m}} e^{-i k_{m}(r-z)}\right\}
\end{aligned}
$$

Following the same procedure used in deriving equation (8), the phase and attenuation can be averaged over a thin slab to obtain

$$
i \Delta \bar{\phi}_{m}(z+\Delta z)-\Delta \bar{q}_{m}(z+\Delta z)=-i \frac{2 \pi}{k_{m}} N(z) A_{m}(z) \Delta z
$$

This result can be converted to two differential equations which can be integrated to obtain equations (14) and (15).

Next, following the same procedure used in deriving equation (24), we obtain the squared deviations

$$
\Delta \overline{\left|\phi_{m}(z+\Delta z)\right|^{2}}+\Delta \overline{\left|q_{m}(z+\Delta z)\right|^{2}}=2 \pi N(z)\left|A_{m}(z)\right|^{2} G_{m} \Delta z
$$

This can be integrated with respect to $z$ to obtain

$$
\overline{\left|\phi_{m}(Z)\right|^{2}}+\overline{\left|q_{m}(Z)\right|^{2}}=2 \pi \int_{0}^{Z} N(z)\left|A_{m}(z)\right|^{2} G_{m} d z
$$


It is also possible to start with equations (C-4) and show that so long as

$$
2 k_{m}(r-z) \gg \beta_{m}
$$

where $\beta_{m}$ is given in Appendix A, the squared fluctuations will be evenly distributed between $\overline{|\phi|^{2}}$ and $\overline{|q|^{2}}$ and thus

$$
\overline{\left|\phi_{m}(Z)\right|^{2}}=\overline{\left|q_{m}(Z)\right|^{2}}=\pi \int_{0}^{Z} N(z)\left|A_{m}(z)\right|^{2} G_{m} d z
$$

\section{References}

1. Aki, K. (1969). Analysis of the seismic coda of local earthquakes as scattered waves, J. Geoph. Res., 74, 615-631.

2. Aki, K. (1973). Scattering of P-waves under the Montana LASA, J. Geoph. Res., 79, 13341336.

3. Aki, K. (1980). Scattering and attenuation of shear waves in the lithosphere, J. Geoph. Res., $85,6469-6504$.

4. Chernov, L. A. (1960). Wave Propagation in a Random Medium, English translation by R. A. Silverman, Dover, New York, 168 pp.

5. Dubrovsky, V. A., and Morochnik, V. S. (1986). Scattering of elastic waves from a large spherical low-contrast inclusion, Fizika Zemli, 4, 32-41, (in Russian).

6. Flatté, S. M., and Wu, R. S. (1988). Small-scale structure in the lithosphere and asthenosphere deduced from arrival time and amplitude fluctuations, J. Geoph. Res., 93, 6601-6614.

7. Groenenboom, J., and Snieder, R. (1995). Attenuation, dispersion, and anisotropy by multiple scattering of transmitted waves through distributions of scatterers, J. Acoust. Soc. Am., 98, 3482-3492. 
8. Hudson, J. A. (1968). The scattering of elastic waves by granular media, Q. J. Mech. Appl. Math., 21, 487-502.

9. Hudson, J. A. (1977). Scattering waves in the coda of P, J. Geophys., 43, 359-374, 1977.

10. van de Hulst, H. C. (1957). Light scattering by small particles, John Wiley Inc., New York, $470 \mathrm{pp}$.

11. Kaelin, B., and Johnson, L. R. (1998). Dynamic composite elastic medium theory. Part II. Three-dimensional media, J. Appl. Phys., 84, 5458-5468, 1998.

12. Knopoff, L., and Hudson, J. A. (1964). Scattering of elastic waves by small inhomogeneities, J. Acoust. Soc. Am., 36, 338-343.

13. Knopoff, L., and Hudson, J. A. (1967). Frequency dependence of scattered elastic waves, $J$. Acoust. Soc. Am., 42, 18-20.

14. Korneev, V. A., and Johnson, L. R. (1993a). Scattering of elastic waves by a spherical inclusion, I. Theory and numerical results, Geophys. J. Int., 115, 230-250.

15. Korneev, V. A., and Johnson, L. R. (1993b). Scattering of elastic waves by a spherical inclusion, II. Limitations of asymptotic solutions, Geophys. J. Int., 115, 251-263.

16. Korneev, V. A., and Johnson, L. R. (1996). Scattering of P and S waves by a spherically symmetric inclusion, Pure and Appl. Geophys., 147, 675-718.

17. Kuster, G. T., and Toksöz, M. N. (1974). Velocity and attenuation of seismic waves in two-phase media : Part 1. Theoretical formulations. Geophysics, 39, 587-606.

18. Li, X., and Hudson, J. A. (1995). Elastic scattered waves from a continuous and heterogeneous layer, Geophys. J. Int., 121, 82-102. 
19. Li, X., and Hudson, J. A. (1997). Time-domain computation of scattering from a heterogeneous layer, Geophys. J. Int., 128, 197-203.

20. Sato, H. (1982a). Amplitude attenuation of impulsive waves in random media based on travel time corrected mean wave formalism, J. Acoust. Soc. Am., 71, 559-564.

21. Sato, H. (1982b). Attenuation of $S$ waves in the lithosphere due to scattering by its random velocity structure. J. Geoph. Res., 87, 7779-7785.

22. Sato, H. (1984). Attenuation and envelope formation of three-component seismograms of small local earthquakes in randomly inhomogeneous lithosphere, J. Geophys. Res., 89, 12211241.

23. Sato, H. (1990). Unified approach to amplitude attenuation and coda extension in the randomly inhomogeneous lithosphere, Pure Appl. Geophys., 132, 93-122.

24. Wu, R. S. (1982). Attenuation of short period seismic waves due to scattering, Geoph. Res. Lett., 9, 9-12.

25. Wu, R. S. (1989). The perturbation method in elastic scattering, Pure Appl. Geophys., 131, 605-637.

26. Wu, R. S. (1994). Wide-angle elastic wave one-way propagation in heterogeneous media and an elastic wave complex-screen method, J. Geophys. Res., 99, 751-766.

27. Wu, R. S., and Aki, K. (1985a). Scattering characteristics of elastic waves by an elastic heterogeneity, Geophysics, 50, 582-595.

28. Wu, R. S., and Aki, K. (1985b). Elastic wave scattering by a random medium and the small-scale inhomogeneities in the lithosphere. J. Geoph. Res., 90, 10261-10273. 


\section{Figure Captions}

Figure 1 The basic geometry for the scattering problems considered in this paper. A plane $\mathrm{P}$ or $\mathrm{S}$ wave propagating in the $z$ direction encounters a thin slab of thickness $\Delta z$ containing randomly distributed spherical scatterers having material properties different from the background medium. A distance $h$ on the other side of the slab there is a line of receivers spaced a distance $\Delta x$ apart in the $x$ direction.

Figure 2 Comparison between effective velocities calculated with the low-frequency results from this paper (solid lines) and the effective media results of Kuster and Toksöz (dashed lines). The background medium has properties: $v_{p}=5.3 \mathrm{~km} / \mathrm{sec}, v_{s}=3.2 \mathrm{~km} / \mathrm{sec}, \rho=2.65 \mathrm{gm} / \mathrm{cm}^{3}$. Part (a) is for low velocity scatterers with properties: $v_{p}=3.0 \mathrm{~km} / \mathrm{sec}, v_{\mathrm{s}}=2.0 \mathrm{~km} / \mathrm{sec}, \rho=2.6 \mathrm{gm} / \mathrm{cm}^{3}$. Part (b) is for high velocity scatterers with properties: $v_{p}=7.0 \mathrm{~km} / \mathrm{sec}, v_{s}=4.0 \mathrm{~km} / \mathrm{sec}$, $\rho=3.0 \mathrm{gm} / \mathrm{cm}^{3}$.

Figure 3 Synthetic seismograms for waves that have been transmitted through a slab of thickness $\Delta z=0.5 \mathrm{~km}$ containing spherical scatterers randomly distributed with a concentration $c=0.1$. For this example the background medium has properties: $v_{p 2}=5.3 \mathrm{~km} / \mathrm{sec}, v_{s 2}=3.2 \mathrm{~km} / \mathrm{sec}$, $\rho_{2}=2.65 \mathrm{gm} / \mathrm{cm}^{3}$ while the scatterers have the lower values: $v_{p 1}=3.0 \mathrm{~km} / \mathrm{sec}, v_{s 1}=2.0 \mathrm{~km} / \mathrm{sec}$, $\rho_{1}=2.60 \mathrm{gm} / \mathrm{cm}^{3}$ The results are shown for 20 different values of $x$ where the offset in the $z$ direction is $h=4.0 \mathrm{~km}$ and the spacing in the $x$ direction is $\Delta x=0.5 \mathrm{~km}$. Part (a) is for an incident $\mathrm{S}$ wave and the transmitted waves are shown for the $x$ component. Part (b) is for an incident $\mathrm{P}$ wave and the transmitted waves are shown for the $z$ component.

Figure 4 Scattering characteristics for an incident $S$ wave as calculated by taking Fourier transforms of the seismograms in Figure 3a, where the results for a single seismogram are shown in the 
upper panels ( $a$ and $b$ ) and the results for the average of all the seismograms are shown in the lower panels ( $c$ and d). The results of these transforms are shown as broken lines, while analytical estimates for the mean values are shown as heavy solid lines and analytical estimates.for the mean plus and minus one standard deviation are shown as light solid lines. The nondimensional attenuation is shown on the left ( $a$ and $c$ ) and the nondimensional phase is shown on the right ( $b$ and d).

Figure 5 Similar to Figure 4 for an incident $\mathrm{P}$ wave and the seismograms of Figure $3 \mathrm{~b}$.

Figure 6 Amplitude of the fluctuations as a function of the non-dimensional distance of propagation $c Z / R$, where $R$ is the radius of the scatterers, $c$ their concentration, and $Z$ the distance of propagation. The results are shown for various values of the non-dimensional frequency $W_{m}=\omega R / v_{m 2}$, where $\omega$ is angular frequency, $v_{m 2}$ is the background velocity, and $m$ can be either $p$ for an incident $\mathrm{P}$ wave or $s$ for an incident $\mathrm{S}$ wave. The material properties are the same as those used in Figure 3.

Figure 7 Similar to Figure 6 except that the non-dimensional distance to the maximum in the fluctuation amplitude curve is now plotted as a function of non-dimensional frequency $\omega R / v_{s}$ for both incident $S$ waves (dashed line) and incident $P$ waves (solid line). 
Figure 8 Theoretical effective velocities for the transmitted coherent $\mathrm{P}$ and $\mathrm{S}$ waves as a function of the non-dimensional frequency $\omega R / v_{s}$.

Figure B-1 The functions $g_{k}(w), k=0,1,2,3,4,5,6$ (solid lines) and their approximations (dashed lines). 


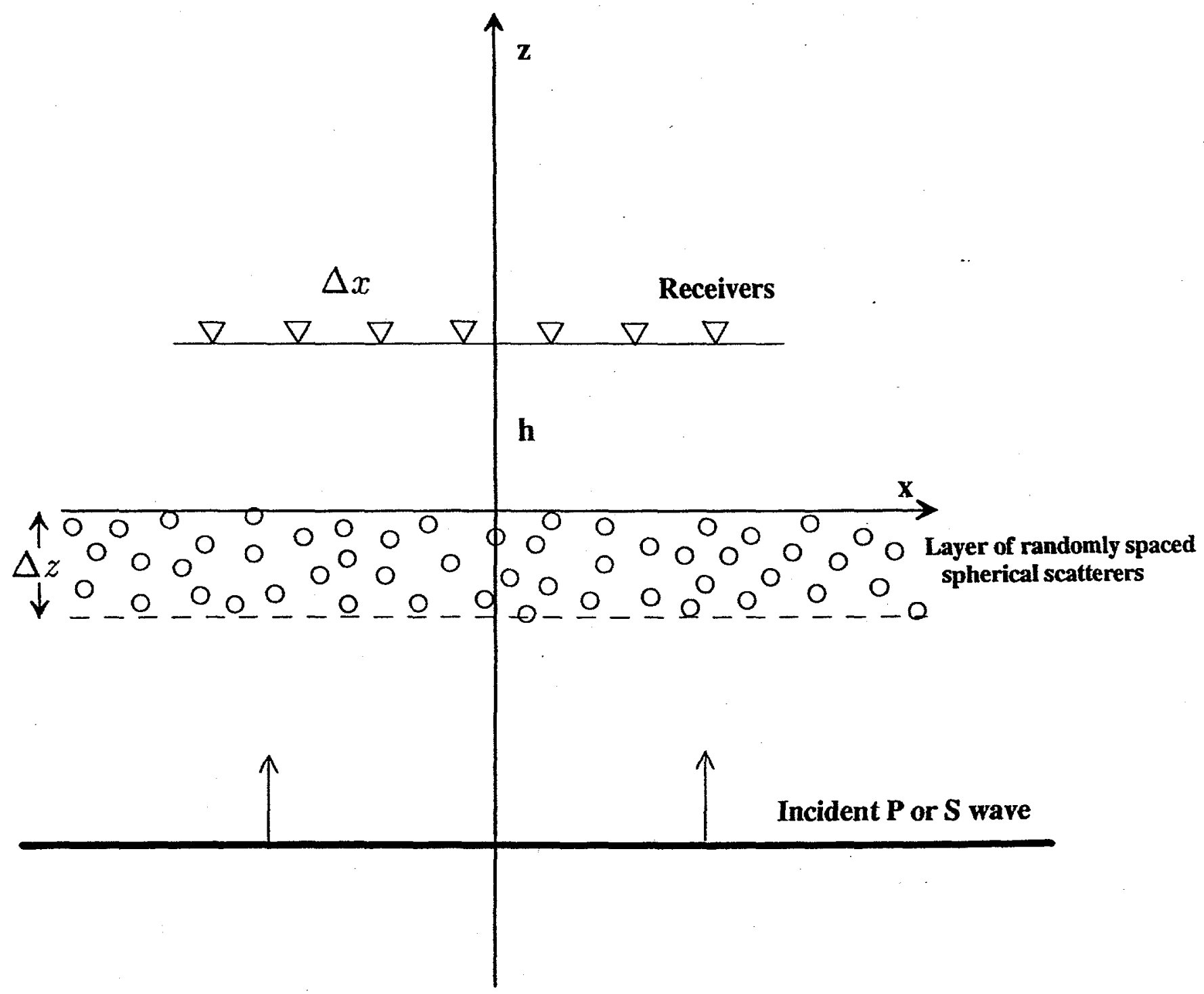

Figure 1 The basic geometry for the scattering problems considered in this paper. A plane $\mathbf{P}$ or $S$ wave propagating in the $z$ direction encounters a thin slab of thickness $\Delta z$ containing randomly distributed spherical scatterers having material properties different from the background medium. A distance $h$ on the other side of the slab there is a line of receivers spaced a distance $\Delta x$ apart in the $x$ direction. 


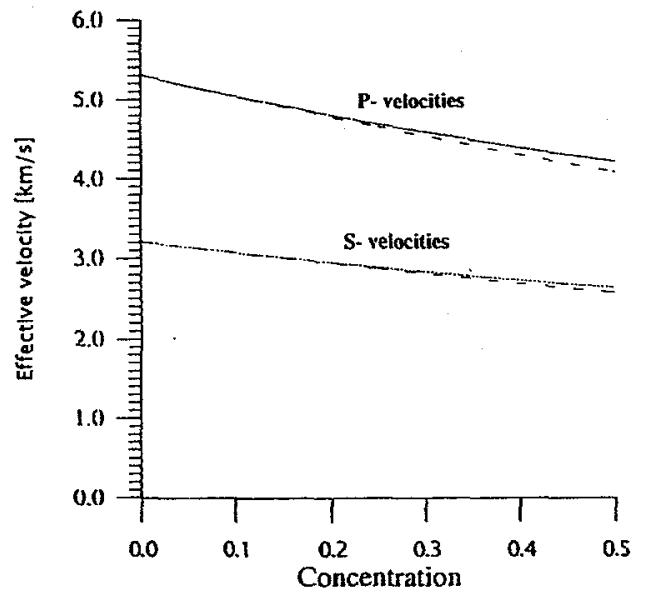

a.

Figure 2 Comparison between effective velocities calculated with the low-frequency results from this paper (solid lines) and the effective media results of Kuster and Toksöz (dashed lines). The background medium has properties: $v_{p}=5.3 \mathrm{~km} / \mathrm{sec}, v_{s}=3.2 \mathrm{~km} / \mathrm{sec}, \rho=2.65 \mathrm{gm} / \mathrm{cm}^{3}$. Part (a) is for low velocity scatterers with properties: $v_{p}=3.0 \mathrm{~km} / \mathrm{sec}, v_{s}=2.0 \mathrm{~km} / \mathrm{sec}, \rho=2.6 \mathrm{gm} / \mathrm{cm}^{3}$. Part (b) is for high velocity scatterers with properties: $v_{p}=7.0 \mathrm{~km} / \mathrm{sec}, v_{s}=4.0 \mathrm{~km} / \mathrm{sec}$, $\rho=3.0 \mathrm{gm} / \mathrm{cm}^{3}$. 


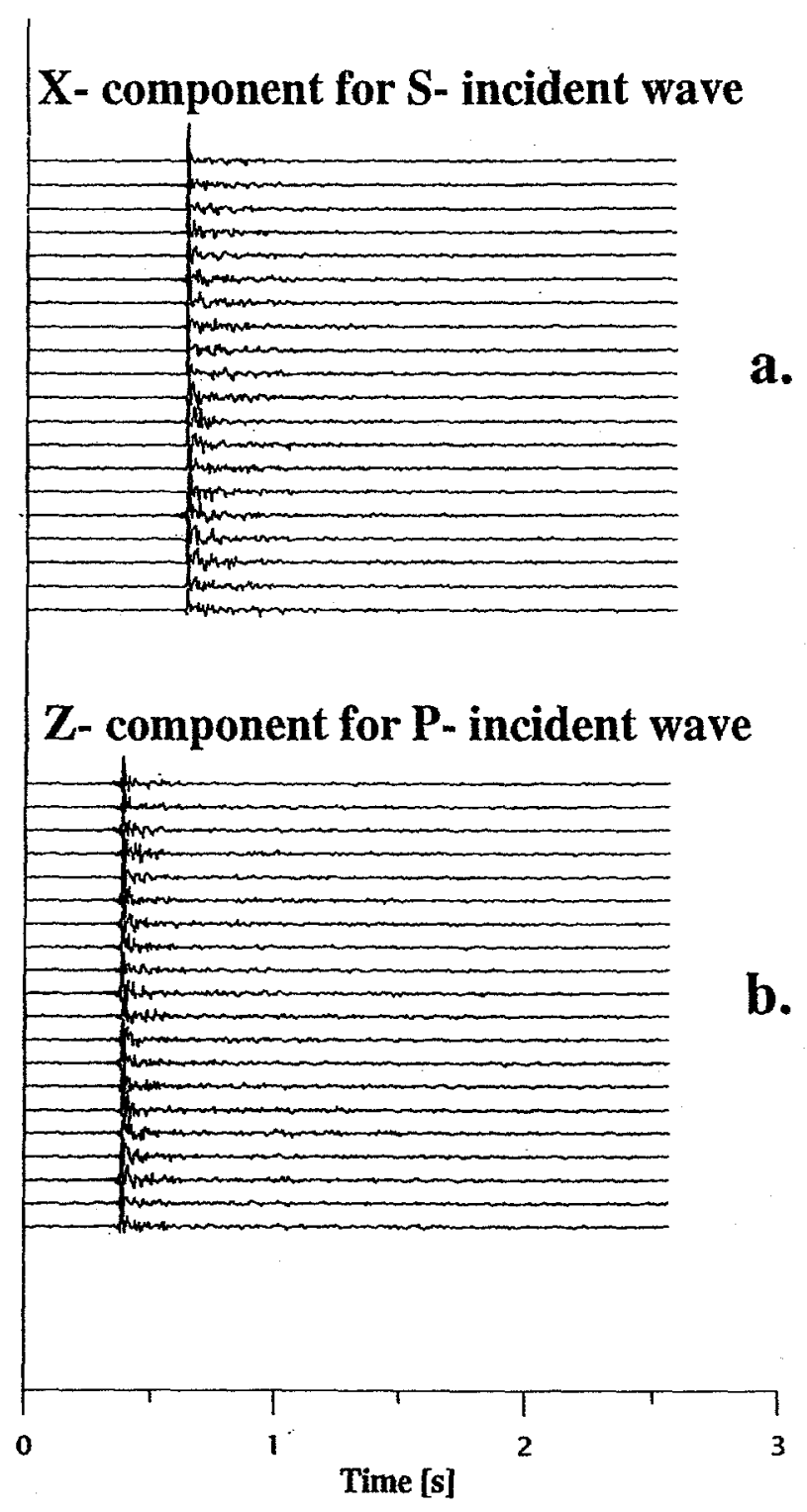

Figure 3 Synthetic seismograms for waves that have been transmitted through a slab of thickness $\Delta z=0.5 \mathrm{~km}$ containing spherical scatterers randomly distributed with a concentration $c=0.1$. For this example the background medium has properties: $v_{p 2}=5.3 \mathrm{~km} / \mathrm{sec}, v_{s 2}=3.2 \mathrm{~km} / \mathrm{sec}$, $\rho_{2}=2.65 \mathrm{gm} / \mathrm{cm}^{3}$ while the scatterers have the lower values: $v_{p 1}=3.0 \mathrm{~km} / \mathrm{sec}, v_{s 1}=2.0 \mathrm{~km} / \mathrm{sec}$, $\rho_{1}=2.60 \mathrm{gm} / \mathrm{cm}^{3}$ The results are shown for 20 different values of $x$ where the offset in the $z$ direction is $h=4.0 \mathrm{~km}$ and the spacing in the $x$ direction is $\Delta x \doteq 0.5 \mathrm{~km}$. Part (a) is for an incident $S$ wave and the transmitted waves are shown for the $x$ component. Part (b) is for an incident $\mathrm{P}$ wave and the transmitted waves are shown for the $z$ component. 

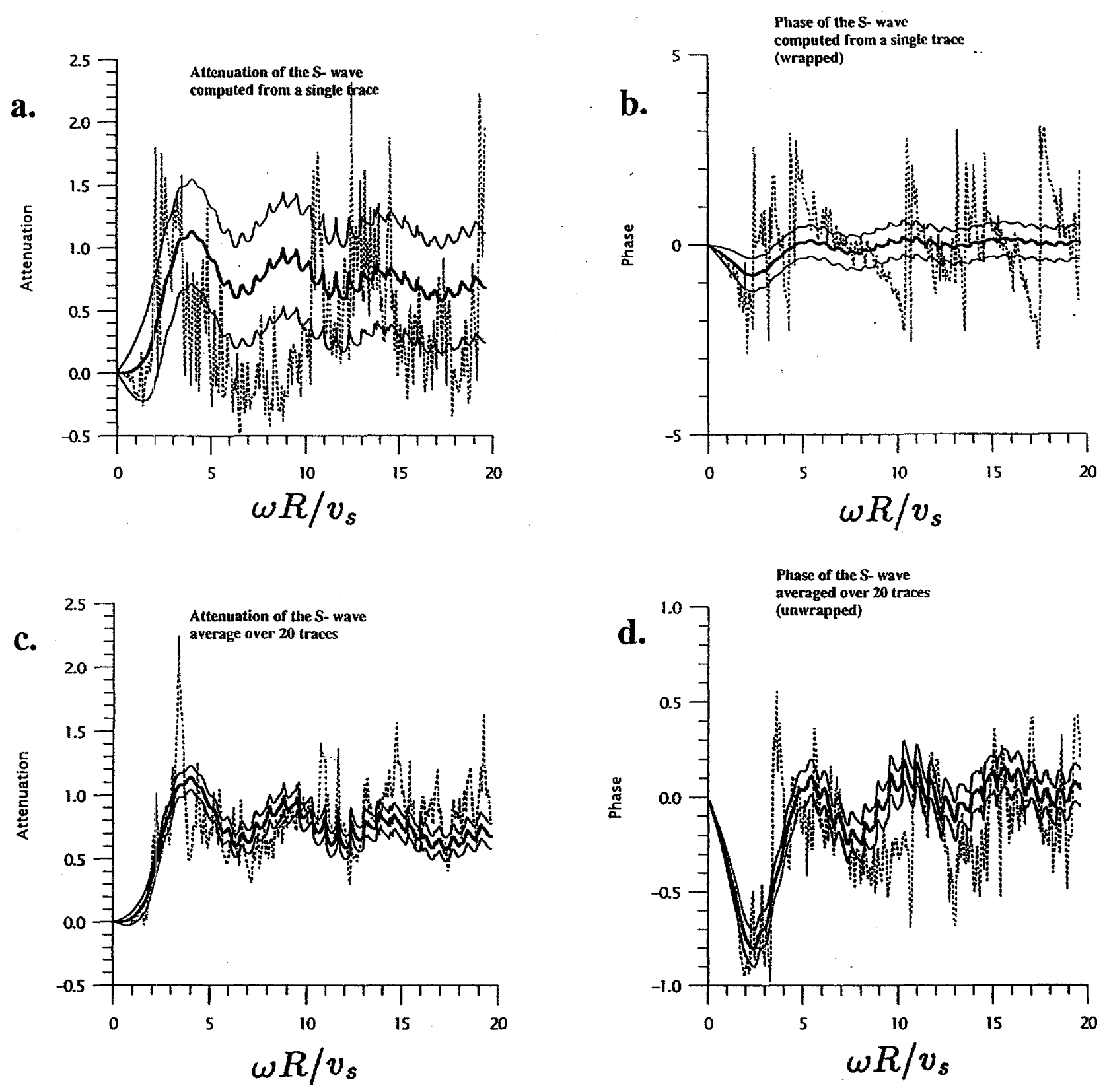

Figure 4 Scattering characteristics for an incident $S$ wave as calculated by taking Fourier transforms of the seismograms in Figure 3a, where the results for a single seismogram are shown in the upper panels ( $a$ and $b$ ) and the results for the average of all the seismograms are shown in the lower panels ( $c$ and $d$ ). The results of these transforms are shown as broken lines, while analytical estimates for the mean values are shown as heavy solid lines and analytical estimates for the mean plus and minus one standard deviation are shown as light solid lines. The nondimensional attenuation is shown on the left ( $a$ and $c$ ) and the nondimensional phase is shown on the right ( $b$ and d). 

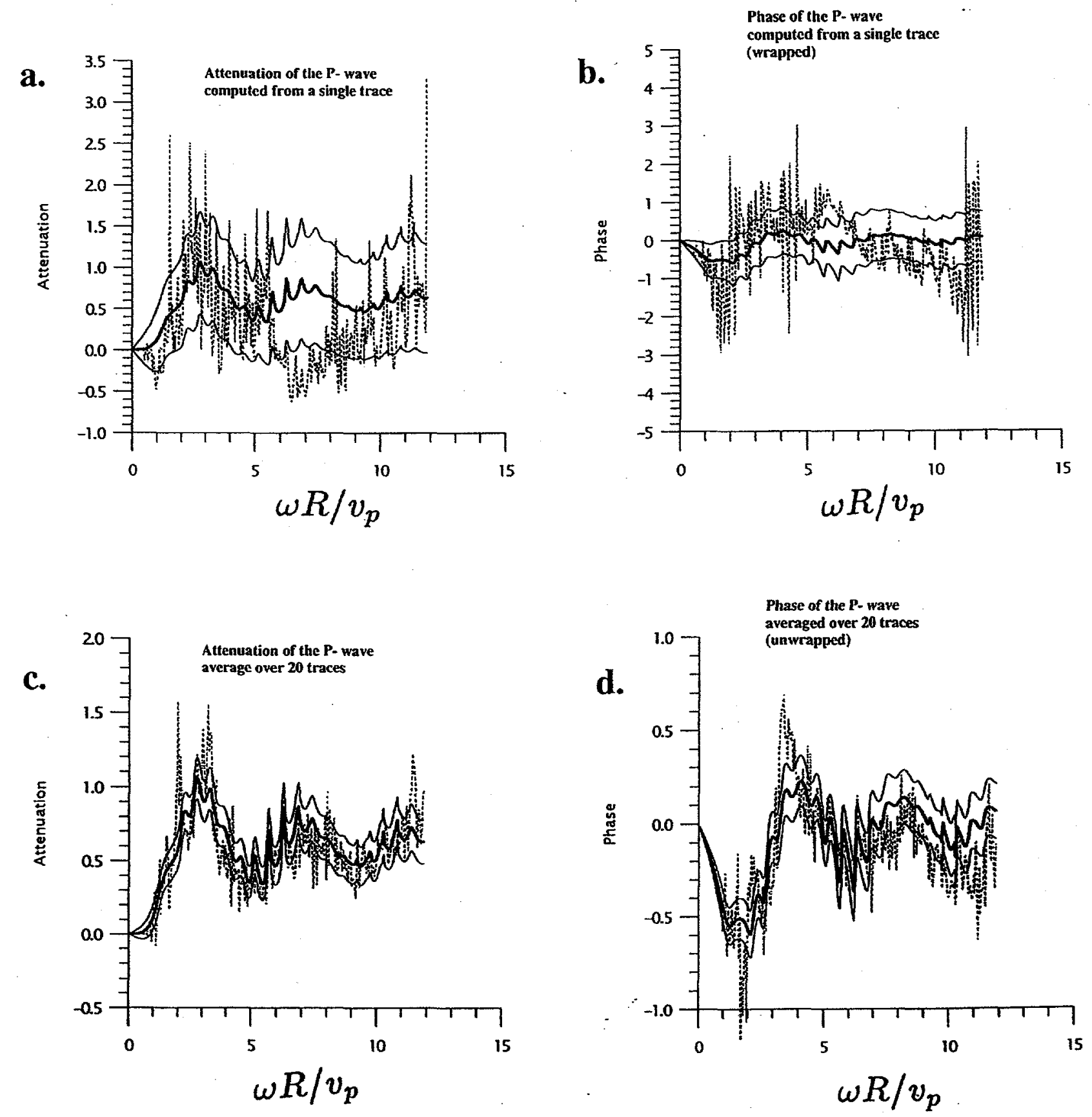

Figure 5 Similar to Figure 4 for an incident $P$ wave and the seismograms of Figure $3 b$. 


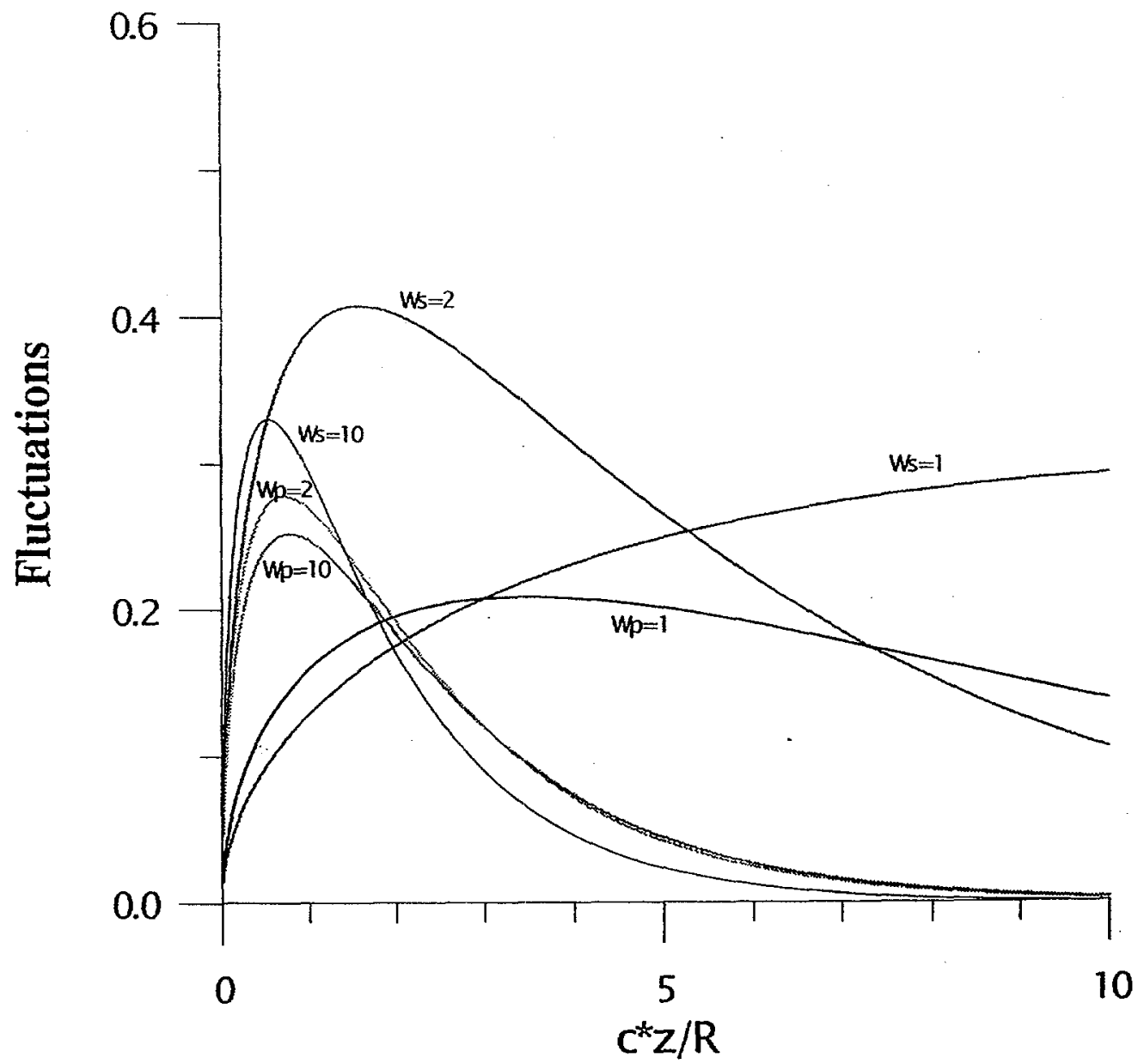

Figure 6 Amplitude of the fluctuations as a function of the non-dimensional distance of propagation $c Z / R$, where $R$ is the radius of the scatterers, $c$ their concentration, and $Z$ the distance of propagation. The results are shown for various values of the non-dimensional frequency $W_{m}=\omega R / v_{m 2}$, where $\omega$ is angular frequency, $v_{m 2}$ is the background velocity, and $m$ can be either $\dot{p}$ for an incident $P$ wave or $s$ for an incident $S$ wave. The material properties are the same as those used in Figure 3. 


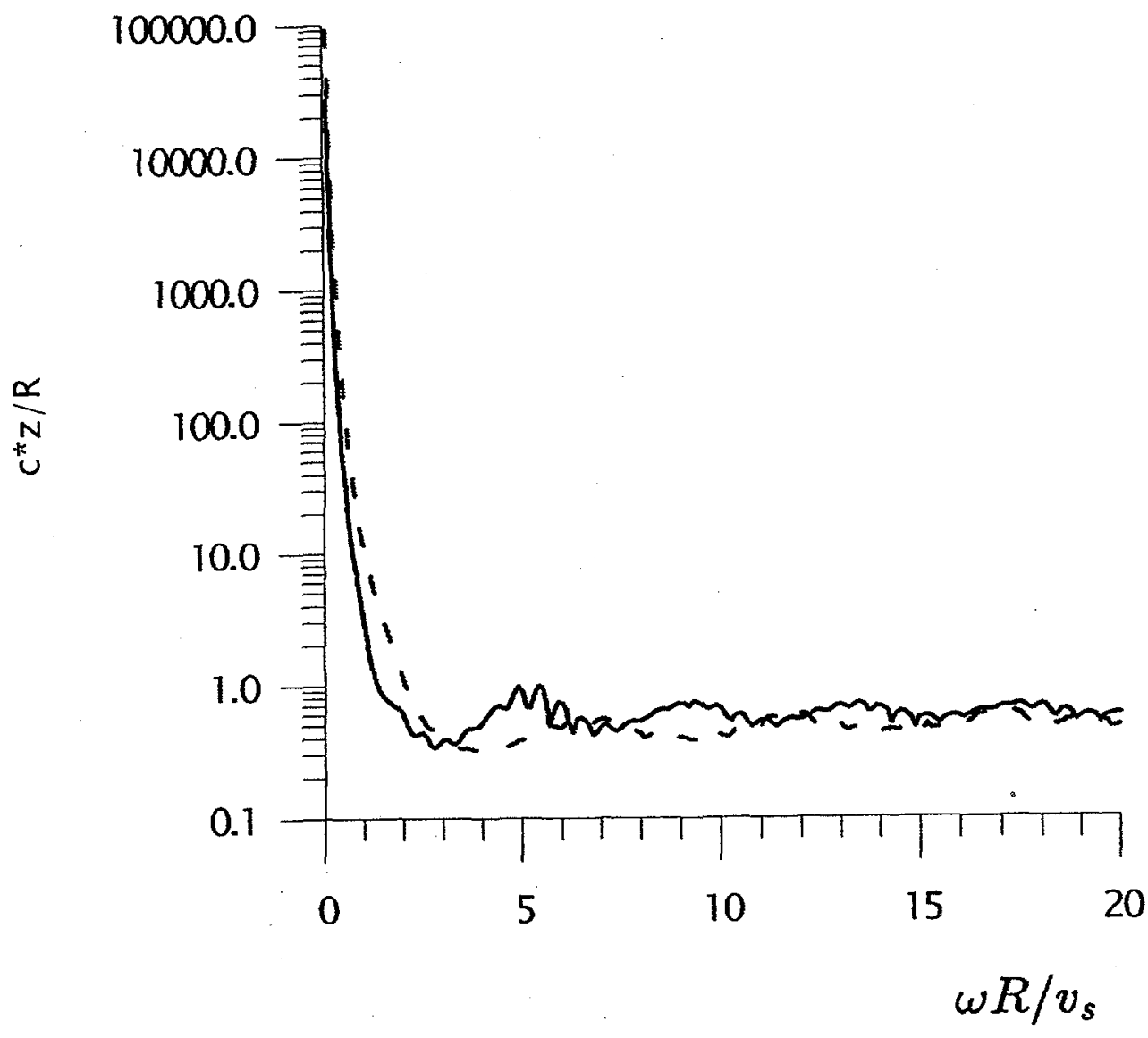

Figure 7 Similar to Figure 6 except that the non-dimensional distance to the maximum in the fluctuation amplitude curve is now plotted as a function of non-dimensional frequency $\omega R / v_{s}$. for both incident $S$ waves (dashed line) and incident $P$ waves (solid line). 


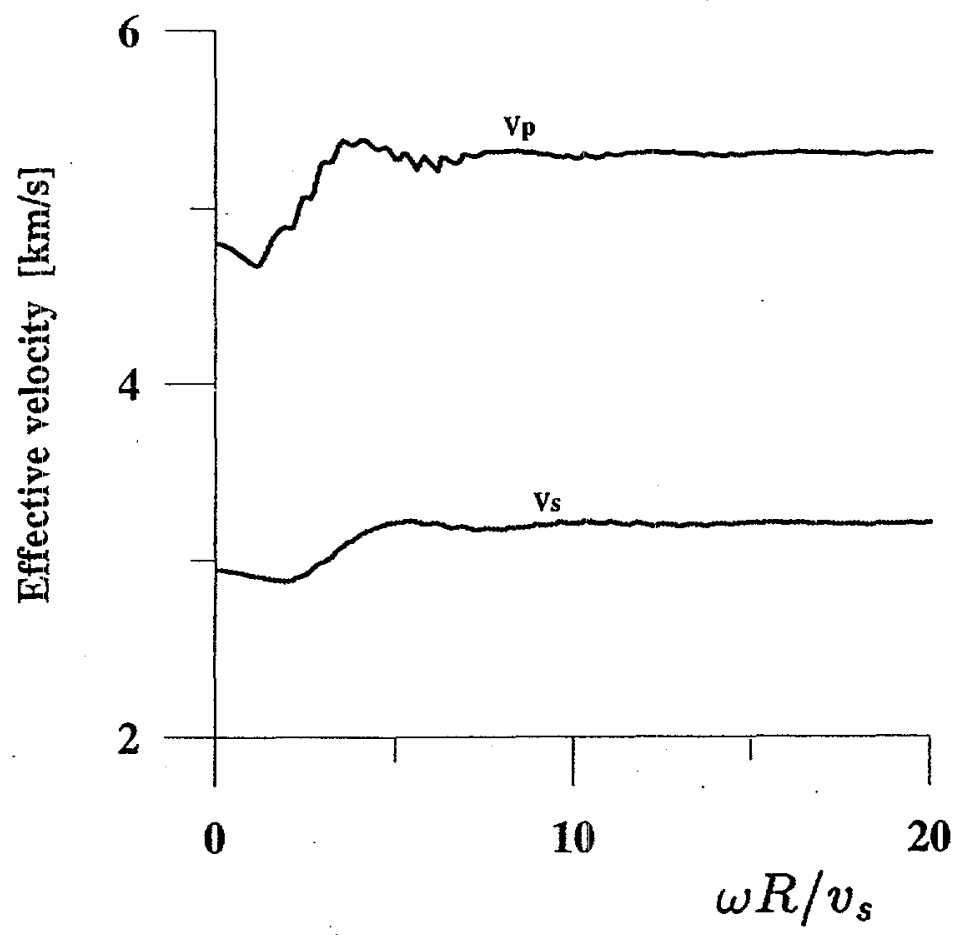

Figure 8 Theoretical effective velocities for the transmitted coherent $\mathrm{P}$ and $\mathrm{S}$ waves as a function of the non-dimensional frequency $\omega R / v_{s}$. 


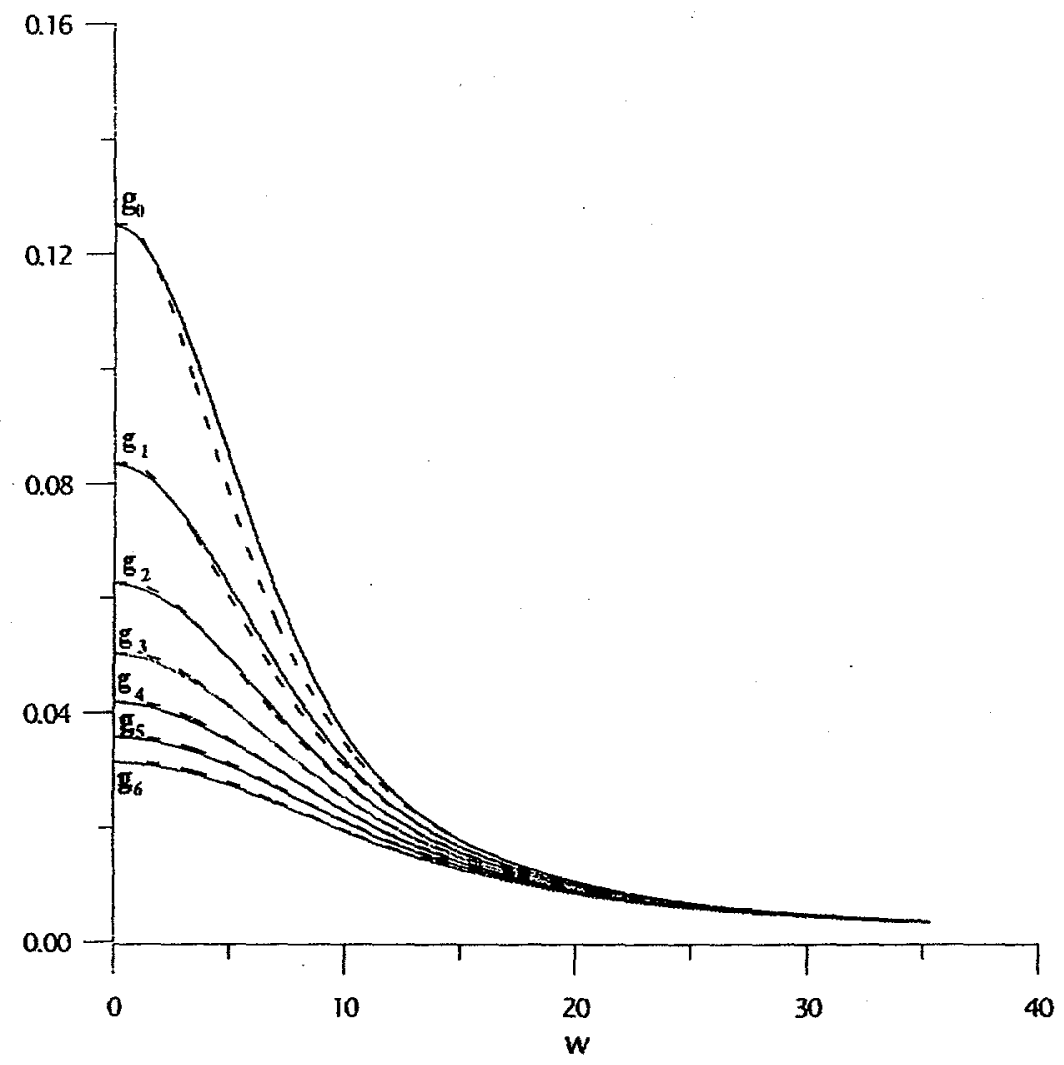

Figure B-1 The functions $g_{k}(w), k=0,1,2,3,4,5,6$ (solid lines) and their approximations (dashed lines). 